\title{
Recent Advances in Feedstock and Lipase Research and Development towards Commercialization of Enzymatic Biodiesel
}

\author{
Vasudeo Zambare ${ }^{1,2}$, Rutuja Patankar ${ }^{3}$ (D), Bhushan Bhusare ${ }^{3}$ and Lew Christopher $4,5, *$ (D) \\ 1 Research and Development Department, Om Biotechnologies, Nashik 422011, India; \\ vasuzambare@gmail.com \\ 2 Aesthetika Eco Research Pvt. Ltd., Nashik 422010, India \\ 3 Department of Life Science, School of Science, Sandip University, Nashik 422213, India; \\ rutujapatankar19@gmail.com (R.P.); bhushanbhusare1990@gmail.com (B.B.) \\ 4 Biorefining World LLC, Rapid City, SD 57702, USA \\ 5 Lee Enterprises Consulting, Inc., Sherwood, AR 72120, USA \\ * Correspondence: 1christo@bio-ref.com
}

check for updates

Citation: Zambare, V.; Patankar, R.; Bhusare, B.; Christopher, L. Recent Advances in Feedstock and Lipase Research and Development towards Commercialization of Enzymatic Biodiesel. Processes 2021, 9, 1743. https://doi.org/10.3390/ pr9101743

Academic Editor:

Chiing-Chang Chen

Received: 25 August 2021

Accepted: 25 September 2021

Published: 29 September 2021

Publisher's Note: MDPI stays neutral with regard to jurisdictional claims in published maps and institutional affiliations.

Copyright: (c) 2021 by the authors. Licensee MDPI, Basel, Switzerland. This article is an open access article distributed under the terms and conditions of the Creative Commons Attribution (CC BY) license (https:/ / creativecommons.org/licenses/by/ $4.0 /)$.

\begin{abstract}
Biodiesel is a biodegradable, renewable, and carbon-neutral alternative to petroleum diesel that can contribute to the global effort of minimizing the use of fossil fuels and meeting the ever-growing energy demands and stringent environmental constraints. The aim of this work was to (1) review the recent progress in feedstock development, including first, second, third, and fourth-generation feedstocks for biodiesel production; (2) discuss recent progress in lipase research and development as one of the key factors for establishing a cost-competitive biodiesel process in terms of enzyme sources, properties, immobilization, and transesterification efficiency; and (3) provide an update of the current challenges and opportunities for biodiesel commercialization from techno-economic and social perspectives. Related biodiesel producers, markets, challenges, and opportunities for biodiesel commercialization, including environmental considerations, are critically discussed.
\end{abstract}

Keywords: biodiesel; feedstock; lipase; transesterification; commercialization

\section{Introduction}

Biodiesel is gaining acceptance as a substitute for petrodiesel due to its renewability, biodegradability, recyclability, and carbon neutrality. Major economic and environmental factors drive the broader use of biodiesel, such as growing demand, fast drop-off supply and price hikes of crude oil, depleting oil reserves, air pollution, and increased levels of greenhouse gases (GHG) emissions [1,2]. For the reasons above, biodiesel production in the last decade has increased at an annual growth rate of $11.4 \%$, which is higher than for any other biofuel such as bioethanol, biobutanol, bio-2,5-dimethylfuran biomethanol, renewable aviation, and biojet fuels $[3,4]$.

Owing to its lower density $\left(860-900 \mathrm{~kg} / \mathrm{m}^{3}\right)$, the energy per gallon biodiesel is about 10\% lower (118,000 British Thermal Unit, BTU/gallon) than petrodiesel (130,000 BTU/gallon). However, there are several advantages of biodiesel that make it a competitive, renewable alternative to petrodiesel. Biodiesel can be blended at any level with petrodiesel and can be used in any diesel engine with only minor engine modifications. These modifications that mainly concern the fuel pump design and related injection timing are needed to address some limitations of biodiesel, such as cold starting, engine clogging, and storage [5]. Biodiesel has a higher flashpoint and cetane number than diesel and the emissions from burning biodiesel in a conventional diesel engine contain lower levels of particulate matter, sulfur oxides, hydrocarbons, carbon monoxide, carbon dioxide, and odor [6]. Overall, biodiesel emits 3-fold lower amounts of GHG than petrodiesel and 85\% ethanol-gasoline fuel (E85), and 4.5-times 
lower than gasoline [7]. Biodiesel has better lubricating properties compared to diesel fuels, which increases the usable life of high-pressure fuel injection equipment that relies on the fuel for its lubrication [8]. Furthermore, biodiesel is safer than petrodiesel because it is less combustible and, therefore, causes less damage if spilled or released to the environment [9].

Biodiesel is typically produced by reacting vegetable oils or animal fats with methyl alcohol in the presence of a chemical (alkali, acid) or enzymatic catalyst. The transesterification process produces biodiesel, fatty acid methyl esters (FAME), and glycerol as a co-product $[10,11]$. Biodiesel (B100) is marketed as a fuel, whereas glycerol can be used as an ingredient for making soaps, detergents, shampoos, and cosmetics. Vegetable oils such as sesame seed oil [12], palm oil [13], white mustard oil [14], and soybean oil [15] that consist of long chain of fatty acids have been all used for biodiesel production. In addition, waste biomass, microalgae, waste cooking oils, oily sludge, meat processing waste, and animal fat waste from slaughterhouses $[16,17]$ are all gaining importance as inexpensive feedstock. Related information on these feedstocks is also helpful in the design of strategies for low-cost biodiesel production that can at the same time address waste disposal problems related to waste diversion, recycle and re-use [18]. The direct use of vegetable oils has been controversial because of the elevated viscosity $[19,20]$. For this reason, strategies have been designed to minimize the vegetable oil viscosity, such as dilution, microemulsion, pyrolysis, and transesterification [21].

The different routes for biodiesel production are homogeneous catalysis, heterogeneous catalysis, enzymatic, non-catalytic supercritical, hydro-esterification, microwave, and ultrasound. The most cost-efficient method for the production of biodiesel with higher quality is transesterification of vegetable oils and animal fats using chemical or enzymatic catalysts [22]. The enzymatic catalysis of biodiesel production offers some apparent advantages over the chemical method, which include: room-temperature reaction conditions, elimination of treatment costs associated with the recovery of chemical catalysts, enzyme reuse, high substrate specificity, the ability to convert both free fatty acids and triglycerides to biodiesel in one step, lower alcohol to oil ratio, avoidance of side reactions and minimized impurities, easier product separation and recovery; biodegradability and environmental acceptance [23-27].

In order to extend the viability of the enzymatic biodiesel process, major efforts have been recently made towards process improvement in terms of biodiesel efficiency, the impact of water and fatty acid content, adding value to glycerol co-product and bioreactor design [28]. Research has focused on optimizing process parameters that: (1) directly impact biodiesel production (e.g., oil to alcohol ratio, lipid content, need for treatment and purification, etc.); and (2) have the potential to reduce the number of unit operations further and, consequently, the overall cost of biodiesel production [29]. The success and efficiency of the enzymatically catalyzed biodiesel production are dependent on many factors, such as feedstock, pretreatment, enzyme properties, temperature, time, mixing speed, etc. $[17,30]$ The focus of this review is on the choice of feedstock and enzyme catalyst as two of the major factors affecting the production of enzymatic biodiesel and its properties. Related producers, markets, challenges, and opportunities for biodiesel commercialization, including environmental considerations, are critically discussed.

\section{Feedstock for Biodiesel Production}

The feedstock and its availability as a raw material is a prerequisite for the successful establishment of a large-scale biodiesel production process [31]. While there has been considerable growth in the biodiesel industry, the feedstock availability has acted as a natural barrier that applied pressure on biodiesel producers and impeded the biodiesel scale up. Feedstock cost and supplies are a function of various factors, including currency strength, domestic and global production capacity for biofuels, energy costs, global demand for food, etc. It has been reported that oilseed crops are only capable of meeting a limited biodiesel demand [32]. This illustrates the need for more viable alternative biodiesel feedstocks such as low-cost waste and non-edible oils. In addition, oleaginous microorganisms such as 
algae [33], yeast [34], and bacteria [35] are aggressively studied as promising feedstocks for biodiesel that can help reduce the feedstock shortage [36].

The total cost of producing biodiesel varies and depends on a number of factors, including production method, production scale, quality, and trade cost of raw materials used. Biodiesel production has to look after the investment and operating costs [37]. In terms of availability and cost, the feedstock is a major issue for biodiesel production. Currently, high-quality food-grade vegetable oils, such as soybean, rapeseed, palm, and groundnut oil, are utilized in biodiesel manufacturing. These feedstock expenses account for more than $60 \%$ of the overall biodiesel production costs [38]. Thus, to reduce the cost of biodiesel, many low-cost feedstocks and many alternative techniques need to be explored.

Depending upon the source and processing techniques used, the biodiesel feedstocks are grouped into first, second, third, and fourth generations. The first-generation feedstock implies the direct use of food crops, such as corn, soybean, sugarcane, etc., for biodiesel production [39]. The second-generation feedstock includes non-edible raw materials that are no longer suitable for human consumption. Examples include waste vegetable oils and animal fats. Third-generation feedstock comprises high oil yield producing microalgae [40]. Solar energy-based technology of photosynthetic water splitting as a photobiological solar fuel system represents the fourth-generation feedstock of the future. Table 1 presents examples of all four generation feedstocks and their fatty acid composition.

Table 1. Biodiesel feedstocks and their fatty acid composition.

\begin{tabular}{|c|c|c|c|}
\hline Generation & Feedstock & Fatty Acid Composition & References \\
\hline \multirow{11}{*}{ 1st } & Rapeseed & $\begin{array}{l}\text { Monounsaturated fatty acids (Oleic acid + Alpha } \\
\text { Lipoic Acid + Linoleic acid) }\end{array}$ & [41] \\
\hline & Olive oil & $\begin{array}{c}\text { Monounsaturated fatty acids (Oleic acid + Alpha } \\
\text { Lipoic Acid + Linoleic acid) }\end{array}$ & [41] \\
\hline & $\begin{array}{c}\text { Tea Seed } \\
\text { (Camellia Sinensis) }\end{array}$ & $\begin{array}{c}\text { Palmitic acid + Stearic acid + Oleic acid } \\
+ \text { Linoleic acid + Linolenic acid + Gadoleic acid }\end{array}$ & [42] \\
\hline & Groundnut & $\begin{array}{l}\text { Monounsaturated fatty acids (Saturated fatty } \\
\text { acids + Linoleic acid) }\end{array}$ & [41] \\
\hline & Amaranth seeds & Monounsaturated fatty acids & [43] \\
\hline & Grapeseed & Polyunsaturated fatty acid (Linoleic acid) & [41] \\
\hline & Sesame & $\begin{array}{l}\text { Polyunsaturated fatty acid (Linoleic acid } \\
+ \text { Monounsaturated fatty acids) }\end{array}$ & [41] \\
\hline & Sunflower oil & $\begin{array}{l}\text { Saturated fatty acids + Monounsaturated fatty } \\
\text { acids + Polyunsaturated fatty acid }\end{array}$ & [41] \\
\hline & Okra (Hibiscus esculentus) seed & $\begin{array}{c}\text { Behenic acid + Arachidic acid + Linoleic acid } \\
+ \text { Oleic acid + Stearic acid + Margaric acid } \\
+ \text { Palmitoleic acid + Palmitic acid + Myristic acid }\end{array}$ & [44] \\
\hline & & $\begin{array}{l}\text { Rapeseed Palmitic acid + Stearic + Oleic } \\
+ \text { Linoleic + Linolenic }\end{array}$ & [45] \\
\hline & Depot margarine & Polyunsaturated fatty acid + saturated fatty acid & [46] \\
\hline
\end{tabular}


Table 1. Cont.

\begin{tabular}{|c|c|c|c|}
\hline Generation & Feedstock & Fatty Acid Composition & References \\
\hline \multirow{10}{*}{$\begin{array}{l}\text { 2nd } \\
\text { Plants }\end{array}$} & Jatropha tree (Jatropha curcas) & Palmitic acid + Oleic acid + Alpha lipoic Acid & [47] \\
\hline & Karanja (Pongamia pinnata) & $\begin{array}{c}\text { Oleic acid + Linoleic acid + Palmitic acid + } \\
\text { Stearic acid }\end{array}$ & [48] \\
\hline & Mahua (Madhuca indica) & $\begin{array}{c}\text { Oleic acid }+ \text { Palmitic acid }+ \text { Stearic acid }+ \text { Lipoic } \\
\text { Acid }+ \text { Adipic acid }\end{array}$ & [49] \\
\hline & $\begin{array}{l}\text { Castor bean seed (Ricinus } \\
\text { communis) }\end{array}$ & $\begin{array}{c}\text { Palmitic acid + Oleic acid + Pentanoic acid + } \\
\text { Octanoic acid + Ricinoleic acid }\end{array}$ & {$[50]$} \\
\hline & Neem (Azadirachta indica) & $\begin{array}{c}\text { Lipoic + Oleic acid + Oleic acid + Palmitic acid + } \\
\text { Arachidic acid + Behenic acid + Lignoceric acid + } \\
\text { Palmiticoleic acid }\end{array}$ & [51] \\
\hline & $\begin{array}{l}\text { Salicornia begelovii (dwarf } \\
\text { saltwort) seed }\end{array}$ & $\begin{array}{c}\text { Linoleic acid + palmitic acid + oleic acid + stearic } \\
\text { acid + Linolenic acid }\end{array}$ & [52] \\
\hline & Nagchampa tree & $\begin{array}{c}\text { Linoleic acid + Oleic acid + Stearic acid }+ \\
\text { Palmitic acid }\end{array}$ & [53] \\
\hline & $\begin{array}{l}\text { Rubber seed tree (Hevea } \\
\text { brasiliensis) }\end{array}$ & Oleic acid + Linoleic acid + Linolenic acid & [54] \\
\hline & Tobacco seed (Nicotiana tabacum) & Palmitic acid + Oleic acid & [55] \\
\hline & $\begin{array}{l}\text { Meadowfoam (Limnanthes alba L.) } \\
\text { seed }\end{array}$ & $\begin{array}{l}\text { Eicosenoic acid }+ \text { Docosadienoic acid }+ \text { Erucic } \\
\text { acid }\end{array}$ & [56] \\
\hline \multirow{4}{*}{ Waste oil } & (Bakery) Depot margarine & $\begin{array}{l}\text { Saturated fatty acid + Monounsaturated fatty } \\
\text { acids }\end{array}$ & [46] \\
\hline & $\begin{array}{l}\text { Sunfoil (triple refined sunflower } \\
\text { oil from Restaurant) }\end{array}$ & $\begin{array}{l}\text { Saturated fatty acid }+ \text { Monounsaturated fatty } \\
\text { acids }\end{array}$ & [46] \\
\hline & Frying oil & $\begin{array}{l}\text { Palmitic acid + Stearic acid + Oleic acid + } \\
\text { Linoleic acid + Linolenic acid }\end{array}$ & [57] \\
\hline & Waste activated sludge & $\begin{array}{l}\text { Palmitic acid + heptadecanoic acid + ginkgoid } \\
\text { acid + stearic acid + oleic acid + linoleic acid }\end{array}$ & [58] \\
\hline \multirow{6}{*}{ Animal oil/fat } & Pork Lard & $\begin{array}{c}\text { Myristic + palmitic + palmitoleic + stearic + oleic } \\
+ \text { linoleic }+ \text { linolenic + Arachidonic }+ \\
\text { docosapentaenoic }\end{array}$ & [59] \\
\hline & Beef Tallow & $\begin{array}{c}\text { Myristic + palmitic + palmitoleic + stearic + oleic } \\
+ \text { linoleic }+ \text { linolenic }\end{array}$ & {$[60]$} \\
\hline & Animal fat & $\begin{array}{c}\text { Myristic + palmitic + palmitoleic + stearic + oleic } \\
+ \text { linoleic + linolenic }\end{array}$ & {$[61]$} \\
\hline & Poultry Fat & $\begin{array}{l}\text { Myristic + palmitic + palmitoleic + stearic + oleic } \\
+ \text { linoleic + linolenic + Arachidonic }+ \\
\text { docosapentaenoic + docosahexaenoic }\end{array}$ & {$[62]$} \\
\hline & Tallow & Palmitoleic + oleic + stearic + palmitic + myristic & [63] \\
\hline & Meat Processing Waste & $\begin{array}{l}\text { Myristic + palmitic + palmitoleic + stearic + oleic } \\
+ \text { linoleic + linolenic + eicosadienoic + saturated } \\
\text { fatty acids }\end{array}$ & [64] \\
\hline \multirow[t]{2}{*}{3 rd } & $\begin{array}{c}\text { Cyanobacteria } \\
\text { (Fremyella diplosiphon) }\end{array}$ & $\begin{array}{l}\text { Methyl palmitate + hexadecanoic acid + methyl } \\
\text { dodecanoate + methyl myristate }+ \\
\text { hexadecenoate + octadecanoate + octadecenoate } \\
+ \text { octadecadienoate }\end{array}$ & [35] \\
\hline & Algae & Palmitic + stearic + oleic + linoleic & [45] \\
\hline
\end{tabular}


Table 1. Cont.

\begin{tabular}{|c|c|c|c|}
\hline Generation & Feedstock & Fatty Acid Composition & References \\
\hline \multirow{5}{*}{ 4th } & Dunaliella tertiolecta & - & [65] \\
\hline & $\begin{array}{c}\text { Marine alga Nannochloropsis } \\
\text { oceanica + oleaginous fungus } \\
\text { Mortierella elongata }\end{array}$ & - & [66] \\
\hline & Phaeodactylum tricornutum & $\begin{array}{l}\text { Saturated fatty acid + monounsaturated fatty } \\
\text { acids }+ \text { polyunsaturated fatty acid }\end{array}$ & [67] \\
\hline & $\begin{array}{l}\text { Phaeodactylum tricornutum Pt4 } \\
\text { Co-expression of a yeast } \\
\text { diacylglycerolacyl-transferase } \\
\text { (ScDGA1) and a plant oleosin } \\
\text { (At-OLEO3) }\end{array}$ & - & [68] \\
\hline & $\begin{array}{l}\text { Chlamydomonas reinhardtii with } \\
\text { overexpressing a Dof-type } \\
\text { transcription factor }\end{array}$ & - & [69] \\
\hline
\end{tabular}

Bio-based feedstocks, including oils (vegetable, algal, microbial) and animal fats, are considered a potent and promising renewable source for biodiesel production. Depending on the feedstock composition, biodiesel with different degrees of purity and properties can be produced [27]. The feedstock selection, therefore, has a direct impact on the choice of the bioprocessing method used, the biodiesel yield, and the cost.

The biodiesel feedstocks are categorized into edible, non-edible, and waste-based [19]. The feedstock availability and selection for biodiesel production are country and regionspecific [70]. For instance, biodiesel production in Canada is based on canola oil, whereas soybean meal is the feedstock of choice in the USA and Brazil. European countries (Italy, Finland, Germany, and UK) predominantly use rapeseed oil as biodiesel feedstock. The abundance of coconut and palm oil dictates the biodiesel production from these feedstocks in Indonesia and Malaysia, respectively [15,71,72]. In India, karanja and jatropha oils are the main biodiesel feedstock $[73,74]$. The growth of non-edible feedstocks for biodiesel production is gaining momentum. China, for instance, has recently set aside a region just to grow jatropha and other non-food oil plants, whereas India has around 60 million hectares of non-cultivated land, which may be used for jatropha production [75].

\subsection{First Generation Feedstock}

Among the edible vegetable oils, sunflower, rapeseed, soybean, and mustard oil are all first-generation feedstock. However, due to food security issues, biodiesel commercialization from edible oils is a challenging one [76]. Small-scale biodiesel production at a farm level utilizes first-generation feedstocks, such as coconut [77], palm tree [78], soybean [79], and sunflower [80]. Some added advantages of first-generation feedstocks are their abundance, availability, biodegradability, easy production in available infrastructure, and technology [81]. However, the major constraints in the production of first-generation biodiesel are the feedstock price and availability, limited production capacity, and competition with food production.

\subsection{Second Generation Feedstock}

The application of second-generation feedstocks from non-edible oil sources (waste cooking oil, tallow oil, animal fats, fish oil, etc.) avoids the ongoing food vs. fuel conflict, with no direct effect on the food supply chain and added benefits of biodegradability, low sulfur and aromatic content $[82,83]$. Although the second-generation feedstocks are mainly low-value non-edible oils, their processing to biodiesel may add extra cost and time. Non-edible feedstocks, also called energy crops, include: jatropha [84-87], jojoba [88], tobacco seed [89], salmon oil [90], and sea mango [91]. Biodiesel generated through energy 
crops is a clean alternative fuel to petrodiesel; however, the supply of this feedstock in large quantities is unfortunately not sustainable [92]. Additionally, the cooking waste oils, restaurant grease, and animal fats [93], such as beef tallow and pork lard [94], are also considered second-generation feedstocks. Non-edible oils crops could be grown on wasteland for maximum utilization of the country's resources. Because of limited availability, however, the second-generation feedstocks cannot fulfill the transportation fuel demand alone. Moreover, biodiesel generated from non-edible vegetable oils and animal fats showed poor engine performance in cold environments due to their high concentration of saturated FAME that is known to have inferior cold flow properties, namely cloud and pour point [95]. For example, the undesirable high melting point and high viscosity of beef tallow (a second-generation feedstock) are due to the presence of nearly $50 \%$ saturated fatty acids from the total amount of fatty acid in beef tallow [94]. The cold flow of properties of biodiesel containing high levels of saturated FAME can be improved through winterization, adding fuel additives or branched branched-chain esters, blending with petrodiesel or vegetable oils of lower crystallization temperature, etc. [96]. These additional processing steps, however, render the production economics of biodiesel less attractive, with a low to moderate commercialization potential. Therefore, due to efficiency and sustainability issues, the large-scale biodiesel production from firstand second-generation feedstocks has faced a major hurdle of high biodiesel cost $[97,98]$. A recent development of a second-generation feedstock is fat, oil, and grease (FOG) that is recovered from wastewater discharges from restaurants, kitchens, food processing plants, and slaughterhouses [99]. FOG is a potential biodiesel feedstock due to its lower price, better oxidative stability, flash point, cetane number, and total emissions compared to other feedstocks, and reduced carbon footprint that is realized through wastewater management [100].

\subsection{Third Generation Feedstock}

The third-generation feedstock is of microbial origin and includes oleaginous microalgae, bacteria, fungi, and yeast. The cultivation and production of these oil-generating microorganisms require no land or special growth supplements [101]. The third-generation feedstocks have higher biomass productivity than the conventional crops, which is due to their high photosynthesis efficiency, especially in the photosynthetic microbes [102]. In addition, the oleaginous microbes present a new biodiesel feedstock that overcomes the challenges of availability, adaptability to climatic conditions, and controversy of the food supply chain that are typically faced by the first and second-generation feedstocks [103].

Lipids from microalgal origin appear as the most promising third-generation biodiesel feedstock. Microalgae have very high growth rates and accumulate high levels of lipids (oil) through photosynthesis $[104,105]$. Algal cultivation is easier than plant cultivation. Cultivated algae show fast multiplication [65]. High oil yielding algae can reach up to $70 \%$ oil $w / w$ dry biomass with an annual oil production of $136,900 \mathrm{~L} / \mathrm{ha}$ and biodiesel productivity of 121,104 kg biodiesel/ha [106]. Algal oil-based biodiesel production could become feasible at a large scale provided a continuous supply of feedstock is in place. Hence, algae cultivation or algal farming need to be further explored in both rural and urban areas to ensure feedstock sustainability. This approach could have an added benefit of creating employment opportunities, thereby strengthening the agricultural sector. More research is also needed to address the inherent drawbacks of algal biodiesel associated with its instability and degradability at higher temperatures [107].

\subsection{Fourth Generation Feedstocks}

Considering the feedstock cost for biodiesel development, it is desirable to research and exploit opportunities to develop a very inexpensive, high-energy density feedstock that is easily accessible and of unlimited availability. Solar energy-based technology of photosynthetic water splitting into energy constituents holds promise to become a future biodiesel feedstock [108]. This feedstock could be integrated with a second and or third-generation 
feedstock for a high photon to fuel conversion efficiency (PFCE) using a synthetic biological approach. Thus, the future photobiological solar fuel system will be utilized for the production of high-quality, high-yield, high-efficiency, and high-performance biodiesel. In general, microorganisms can be cultivated in a photobioreactor for a solar-aided fuel conversion in a two-step process that involves microbial biomass production followed by photo-bio-solar fuel production by engineered and immobilized algae or cyanobacteria (Table 1). Current research has focused on achieving a 10\% PFCE that requires prior organism and bioreactor design and construction. Further enhancement of the PFCE may be possible through developing microbial electrosynthesis technologies that utilize electro-biofuel/synthetic-cell hybrid systems as the fourth-generation feedstock [109].

\section{Lipases for Biodiesel Production}

Applications of conventional techniques such as micro-emulsification, pyrolysis, and transesterification with alkaline/acid catalysts are well developed for the processing of biodiesel [21]. While these procedures are widespread and well investigated, there are some pitfalls and technological diffuculties that need to be addressed and overcome, such as catalyst recovery, purity of the glycerol co-product, high energy consumption, significant volumes of wastewater that require treatment, etc. The development and implementation of the lipase enzymatic path have greatly improved the efficiency of biodiesel processing, thus decreasing the size of the process equipment to the microlevel [110]. Lipases are hydrolytic enzymes that catalyze the production of free fatty acids, diacylglycerols, monoacylglycerols, and glycerol by cleaving off the ester bonds of triacylglycerols (TAG) from fats and oils [111]. As lipases possess an unusual trait of hydrolysis over oil-water interfaces, they can also trans-esterify triacylglycerols to fatty acid alkyl esters in the presence of short-chain alcohols, such as methanol and ethanol [112]. Biodiesel production is one of the most significant and spectacular applications of lipases [113]. However, as lipases are ubiquitous in nature, they could find applications in various industries [114]. Their ubiquity is evident from Table 2, which shows that lipases are produced in plants and microorganisms, including bacteria, fungi, and yeasts.

Table 2. Lipase-producing organisms and their habitat.

\begin{tabular}{|c|c|c|c|}
\hline Source & Species & Habitat & Reference \\
\hline \multirow{11}{*}{ Plants } & Triticum aestivum $\mathrm{L}$. & - & [115] \\
\hline & Pachira aquatica & Seed of Tree, UNESP, Brazil & [116] \\
\hline & Coconut (Cocos nucifera linn) seed & $\begin{array}{c}\text { NIFOR substation Abak, Akwa Ibom State of } \\
\text { Nigeria }\end{array}$ & [117] \\
\hline & Castor Beans & Chiltern Seeds (Ulverston, Cumbria, UK) & [118] \\
\hline & $\begin{array}{l}\text { Bay Laurel (Laurus nobilis L.) } \\
\text { Seeds }\end{array}$ & Hatay, Turkey & [119] \\
\hline & $\begin{array}{c}\text { French bean } \\
\text { (Phaseolus vulgaris L.) }\end{array}$ & Landraces of Himachal Pradesh, India & [120] \\
\hline & Nigella sativa L. Seed & Denizli region of Turkey & [121] \\
\hline & Brassica napus L. & $\begin{array}{c}\text { Bangladesh Agriculture Research Institute, } \\
\text { Irshardi, Pabna and Rajshahi Local Shaheb Bazar } \\
\text { Market. }\end{array}$ & [122] \\
\hline & Rice Bran & Bangalore, India & [123] \\
\hline & Jatropha curcas L. & $\begin{array}{c}\text { Isiuwa quarters of the Nigerian Institute for Oil } \\
\text { Palm Research, Benin City, Nigeria }\end{array}$ & [124] \\
\hline & Lupine seeds & Poland & [125] \\
\hline
\end{tabular}


Table 2. Cont.

\begin{tabular}{|c|c|c|c|}
\hline Source & Species & Habitat & Reference \\
\hline \multirow{15}{*}{ Bacteria } & Streptomyces sp. Al-Dhabi-49 & Soil, Saudi Arabia & [126] \\
\hline & $\begin{array}{l}\text { Chryseobacterium polytrichastri } \\
\text { ERMR1:04 }\end{array}$ & Glacier, Sikkim Himalaya & {$[127,128]$} \\
\hline & $\begin{array}{l}\text { Seeds of African oil bean } \\
\text { (Pentaclethra macrophylla Benth) }\end{array}$ & NIFOR, Benin City & [129] \\
\hline & $\begin{array}{l}\text { Chryseobacterium sp. strain IHBB } \\
10212\end{array}$ & Glacier top-surface soil, Himalaya, India & [130] \\
\hline & Bacillus cereus HSS & $\begin{array}{c}\text { Mediterranean Sea, Eastern Harbor, Al Shatby, } \\
\text { and Abu-Qir }\end{array}$ & [131] \\
\hline & Thalassospira permensis M35-15 & Sea water and sediments samples & [132] \\
\hline & Bacillus subtilis strain Kakrayal_1 & Katra region of Jammu and Kashmir, India & [133] \\
\hline & Geobacillus thermoleovorans DA2 & Desert, Southern Sinai & [134] \\
\hline & Pelosinus fermentans & Groundwater, Germany & [135] \\
\hline & Micrococcus luteus & Agriculture field and garden & [136] \\
\hline & Bacillus aerius & Soil and water of hot spring, Shimla & [137] \\
\hline & Ralstonia species & Soil sample, Germany & [138] \\
\hline & Trichoderma harzianum & Soil sample, Turkey & [139] \\
\hline & Acinetobacter baylyi & Marine sludge, Thailand & [140] \\
\hline & Serratia marcescens & Raw milk & [141] \\
\hline \multirow{8}{*}{ Fungi } & Aspergillus fumigatus & $\begin{array}{c}\text { Oil contaminated soil, HRTC workshop, } \\
\text { Himachal Pradesh }\end{array}$ & [142] \\
\hline & Trichoderma reesei strain RF10625 & Fungal Biodiversity Institute, The Netherlands & [143] \\
\hline & Aspergillus niger (strain LFS) & DSM Food Specialties B.V. & [143] \\
\hline & Geotrichum sp. & UNICAMP, Brazil & [144] \\
\hline & Cunninghamella verticillata & Oil-mill waste & [145] \\
\hline & Aspergillus niger & $\begin{array}{c}\text { Dept of Biochemistry and Microbiology, } \\
\text { University of Plovdiv, Bulgaria }\end{array}$ & [146] \\
\hline & Rhizopus chinensis & $\begin{array}{l}\text { (CCTCC) China Center for Type Culture } \\
\text { Collection }\end{array}$ & [147] \\
\hline & Penicillium simplicissimum & Waste from the babassu oil industry & [148] \\
\hline \multirow{3}{*}{ Yeast } & $\begin{array}{l}\text { Limtongella siamensis } \\
\text { DMKU-JMGT1-45 }\end{array}$ & Grease traps, Kasetsart University, Thailand & [149] \\
\hline & Yarrowia lipolytica & Marine oil-contaminated sludge & [150] \\
\hline & Candida rugosa & Sigma-Aldrich Co. (Germany) & [151] \\
\hline
\end{tabular}

\subsection{Lipase Activity and Specificity}

As lipases are obtained from a variety of biological sources (Table 3), they possess diverse substrate and catalytic specificity [152]. The selection of lipase for a specific application must be carried out on the basis of enzyme specificity and stability in various solvents [153]. According to their substrate specificity, lipases are divided into three groups: sn-1,3-specific lipases (hydrolyze ester bonds in the sn-1 and sn-3 position in TAG); sn-2specific lipases (lipases preferentially cleave acyl chains in the sn- 1 and sn-3 position in TAG); and nonspecific lipases $[154,155]$. Lipases with a sn-1,3-regioselectivity are most common, while the sn-2 fatty acids in TAG are less accessible to lipases due to steric hindrances [154]. 
Table 3. Microbial production of lipase on different substrates.

\begin{tabular}{|c|c|c|c|}
\hline Lipase Producer & Lipase Activity & Substrate & Reference \\
\hline Pseudomonas sp. LSK25 & $50.5 \mathrm{U} / \mathrm{mL}$ & $\begin{array}{l}\text { Rice bran oil } \\
\text { Coconut oil }\end{array}$ & [156] \\
\hline Antarctic Pseudomonas sp. & $130.7 \mathrm{U} / \mathrm{mL}$ & Olive oil & [157] \\
\hline Candida viswanathii & $101.1 \mathrm{U} / \mathrm{mL}$ & Olive oil & [158] \\
\hline Pseudomonas sp. LSK25 & 0.35 to $0.4 \mathrm{U} / \mathrm{mL}$ & olive oil & [156] \\
\hline Aspergillus terreus NCFT 4269.10 & $475 \mathrm{U} / \mathrm{mL}$ & Sun flower oil & [159] \\
\hline Bacillus amyloliquefaciens PS35 & $361 \mathrm{mU} / \mathrm{ml}$ & Palm oil & [160] \\
\hline Pseudomonas fluorescens Strain AMS8 & $226.69 \mathrm{U} / \mathrm{mL}$ & Olive oil & [161] \\
\hline Pseudomonas aeuriginosa & $528.54 \mathrm{U} / \mathrm{L}$ & Olive oil & [162] \\
\hline \multirow{5}{*}{ Penicillium camembertii Thom PG-3 } & $422.0 \mathrm{U} / \mathrm{mL}$ & Jojoba oil & \multirow{5}{*}{ [163] } \\
\hline & $92.8 \mathrm{U} / \mathrm{mL}$ & Corn oil & \\
\hline & $128.0 \mathrm{U} / \mathrm{mL}$ & Soybean oil & \\
\hline & $146.5 \mathrm{U} / \mathrm{mL}$ & Rape seed oil & \\
\hline & $180.0 \mathrm{U} / \mathrm{mL}$ & Linseed oil & \\
\hline Colletotrichum gloesporioides 41 & $18.8 \mathrm{U} / \mathrm{mL}$ & Olive oil emulsion & [164] \\
\hline
\end{tabular}

\subsection{Lipase Thermostability and Half-Life}

The enzyme thermostability is an important characteristic that determines the enzyme potential and robustness for industrial applications. Hence, biotechnological research has been focused on the development of thermostable enzymes by 1) genetic engineering, protein engineering, and strain mutation to improve enzyme stability; and 2) bioprospecting and discovery of new thermophilic organisms capable of producing unique thermostable enzymes [165]. For example, a recent study reported the isolation of a novel thermo-halophilic bacteria from a hot spring area in Indonesia. This strain produced a thermostable lipase with a temperature optimum of $70^{\circ} \mathrm{C}$, which was able to catalyze both hydrolysis and transesterification reactions [166].

The half-life of any chemical reaction is defined as the time elapsed to reach half $(50 \%)$ of the initial reactant concentration [167]. The half-life of select lipases and their temperature optima are displayed in Table 4. Strain mutation has been used as a strategy to alter both the half-life and thermostability of produced lipases $[58,165,168]$. Protein engineering of the enzyme structure through introducing a salt bridge in the enzyme macromolecule significantly increased the thermostability of a Stenotrophomonas maltophilia lipase from $40{ }^{\circ} \mathrm{C}$ to $90{ }^{\circ} \mathrm{C}$, with an additional improvement in half-life [165]. Recombinant DNA technology has been applied to enhance lipase production and reduce the overall cost [169].

Table 4. Optimum temperature and half-life of microbial lipases.

\begin{tabular}{|c|c|c|c|c|}
\hline Source & Strain & Optimum Temperature & Half-Life $\left(t^{1} / 2, \min \right)$ & References \\
\hline \multirow{4}{*}{ Geobacillus zalihae } & D43E & \multirow{4}{*}{$70{ }^{\circ} \mathrm{C}$} & 135 & \multirow{4}{*}{ [168] } \\
\hline & $\mathrm{T} 118 \mathrm{~N}$ & & 75 & \\
\hline & E226D & & 165 & \\
\hline & N304E & & 120 & \\
\hline \multirow{5}{*}{ Rhizopus chinensis } & r27RCL & \multirow{5}{*}{$60{ }^{\circ} \mathrm{C}$} & 0.85 & \multirow{5}{*}{ [170] } \\
\hline & $\mathrm{m} 28$ & & 6.5 & \\
\hline & $\mathrm{m} 26$ & & 4.5 & \\
\hline & $\mathrm{m} 28$ & & 6.5 & \\
\hline & $\mathrm{m} 29$ & & 12.3 & \\
\hline
\end{tabular}


Table 4. Cont.

\begin{tabular}{|c|c|c|c|c|}
\hline Source & Strain & Optimum Temperature & Half-Life $(t 1 / 2, \min )$ & References \\
\hline \multirow{2}{*}{ Rhizomucor miehei } & WT RML & & 2.2 & \multirow{2}{*}{ [171] } \\
\hline & M7 & $70{ }^{\circ} \mathrm{C}$ & 27.5 & \\
\hline \multirow{3}{*}{ Penicillium cyclopium } & WT & & 66.7 & \multirow{3}{*}{ [58] } \\
\hline & L41P & $35^{\circ} \mathrm{C}$ & 87.3 & \\
\hline & G47I & & 126 & \\
\hline \multirow{2}{*}{ Rhizopus oryzae } & V209L & & 4.38 & \multirow{2}{*}{ [172] } \\
\hline & D262G & $55^{\circ} \mathrm{C}$ & 4.2 & \\
\hline \multirow{4}{*}{$\begin{array}{l}\text { Geobacillus } \\
\text { thermodenitrificans }\end{array}$} & \multirow{4}{*}{ AV5 } & $50{ }^{\circ} \mathrm{C}$ & 289 & \multirow{4}{*}{ [173] } \\
\hline & & $60^{\circ} \mathrm{C}$ & 208 & \\
\hline & & $70{ }^{\circ} \mathrm{C}$ & 103 & \\
\hline & & $80{ }^{\circ} \mathrm{C}$ & 95 & \\
\hline \multirow{5}{*}{ Bacillus sonorensis } & \multirow{5}{*}{$4 \mathrm{R}$} & $80{ }^{\circ} \mathrm{C}$ & 150 & \multirow{5}{*}{ [174] } \\
\hline & & $90^{\circ} \mathrm{C}$ & 121.59 & \\
\hline & & $100^{\circ} \mathrm{C}$ & 90.01 & \\
\hline & & $110^{\circ} \mathrm{C}$ & 70.01 & \\
\hline & & $120^{\circ} \mathrm{C}$ & 50 & \\
\hline \multirow{5}{*}{ Bacillus sp. } & \multirow{5}{*}{ RSJ-1 } & $55^{\circ} \mathrm{C}$ & 240 & \multirow{5}{*}{ [175] } \\
\hline & & $60{ }^{\circ} \mathrm{C}$ & 150 & \\
\hline & & $65^{\circ} \mathrm{C}$ & 90 & \\
\hline & & $70^{\circ} \mathrm{C}$ & 45 & \\
\hline & & $75^{\circ} \mathrm{C}$ & 30 & \\
\hline Candida antarctica & & $85^{\circ} \mathrm{C}$ & 92 & [176] \\
\hline \multirow{2}{*}{ Burkholderia cepacia } & \multirow{2}{*}{ ATCC 25609} & $50{ }^{\circ} \mathrm{C}$ & 54 & \multirow{2}{*}{ [177] } \\
\hline & & $60^{\circ} \mathrm{C}$ & 46 & \\
\hline Geobacillus sp. & $\mathrm{T} 1$ & $65^{\circ} \mathrm{C}$ & 315 & [178] \\
\hline
\end{tabular}

\subsection{Lipase Reusability}

The harsh conditions of industrial processes require the use of robust enzymes that retain activity over the entire duration of the enzymatic catalysis. Enzyme immobilization on a solid support has been shown to improve the enzyme resistance to denaturation by alcohol and promote enzyme reusability [112,179]. Immobilized enzymes have several advantages over free enzymes, which include prolonged enzyme-substrate contact, enzyme recycling, improved process control, and more efficient product recovery [23].

Enzyme immobilization has revolutionized biocatalysis as it enabled the development and establishment of more cost-efficient biotechnologies that offer higher quality bioproducts. Immobilization methods include physical adsorption, covalent binding, encapsulation, and bio-selective adsorption $[179,180]$. In one study, a lipase enzyme isolated from Arachis hypogaea seeds was immobilized with Ca-alginate and agarose gel, which significantly improved enzyme stability [112]. Another study reported on the immobilization by physical adsorption and entrapment of commercial lipases (Pseudomonas fluoresces AKL, Pseudomonas cepacia PSL, Hog pancreas PHL, Porcine pancreas PPL, and Mucor javanicus MJL) on polyhydroxybutyrate (PHB), sodium alginate and chitosan [179]. Lipase from the marine yeast Yarrowia lipolytica was immobilized on microporous resin, which improved lipase activity over the free enzyme and allowed enzyme re-use [181]. The use of nanotechnology (carbon nanotubes, metal-based nanoparticles, etc.) has become another groundbreaking technology for lipase immobilization [176,182].

\subsection{Lipase-Catalyzed Biodiesel Production}

As can be seen from Table 5, the yield of biodiesel produced by means of lipases varies depending on the lipase source, substrate source, lipase activity, and reaction parameters, such as temperature, time, and alcohol to substrate ratio. Clearly, in each case, optimization 
of the reaction conditions is necessary. Overall, fungi and yeasts have shown strong abilities to produce lipase (Table 5). Bacterial lipases used for biodiesel production have been sourced from Chromobacterium viscosum, Burkholderia cepacia, Enterobacter aerogenes, Thermomyces lanuginosa, Pseudomonas fluorescens, etc. [183].

Table 5. Lipase reaction parameters and biodiesel yield.

\begin{tabular}{|c|c|c|c|c|c|c|c|c|}
\hline $\begin{array}{c}\text { Lipase } \\
\text { Feedstock }\end{array}$ & Lipase Source & Lipase Name & $\begin{array}{c}\text { Lipase Conc. } \\
(\%)\end{array}$ & $\underset{\left({ }^{\circ} \mathrm{C}\right)}{\text { Temp. }}$ & Time (h) & $\begin{array}{c}\text { Molar Ratio } \\
\text { (Alco- } \\
\text { hol/Oil) }\end{array}$ & $\begin{array}{l}\text { Biodiesel } \\
\text { Yield } \\
(\%)\end{array}$ & Reference \\
\hline $\begin{array}{c}\text { Marine } \\
\text { microalga Nan- } \\
\text { nochloropsis }\end{array}$ & $\begin{array}{l}\text { Candida } \\
\text { antarctica }\end{array}$ & $\begin{array}{c}\text { Candida } \\
\text { antarctica } \\
\text { lipase A } \\
\text { (CALA) }\end{array}$ & 10 & 35 & 72 & $8: 1$ & 40.8 & [184] \\
\hline $\begin{array}{l}\text { Microalga } \\
\text { Chlorella } \\
\text { vulgaris }\end{array}$ & $\begin{array}{l}\text { Candida } \\
\text { antarcitica }\end{array}$ & $\begin{array}{c}\text { Lipase B } \\
\text { (Novozyme } \\
435 \text { ) }\end{array}$ & 40 & 40 & 72 & $13: 1$ & 66.7 & [185] \\
\hline $\begin{array}{l}\text { Waste sardine } \\
\text { oil }\end{array}$ & $\begin{array}{c}\text { Aspergillus } \\
\text { niger }\end{array}$ & Lipase & 10 & 30 & 72 & $9: 1$ & 94.5 & [186] \\
\hline Kernel oil & $\begin{array}{l}\text { Thermomyces } \\
\text { lanuginosus }\end{array}$ & Lipozyme TL & 0.25 & 45 & 4.03 & 1.50 & 83.9 & [187] \\
\hline $\begin{array}{c}\text { Chinese Tallow } \\
\text { Kernel oil }\end{array}$ & $\begin{array}{c}\text { Burkholderia } \\
\text { cepacia }\end{array}$ & PS lipase & 20 & 40 & 24 & $4: 1$ & 55.2 & [188] \\
\hline $\begin{array}{l}\text { Soapstock } \\
\text { from rice bran } \\
\text { oil }\end{array}$ & $\begin{array}{l}\text { Candida } \\
\text { antarctica }\end{array}$ & Novozyme 435 & 10 & 40 & 24 & $5: 1$ & 93.0 & [189] \\
\hline $\begin{array}{l}\text { Soapstock } \\
\text { from rice bran } \\
\text { oil }\end{array}$ & $\begin{array}{l}\text { Thermomyces } \\
\text { lanuginosus }\end{array}$ & $\underset{\text { IM }}{\text { Lipozyme TL }}$ & 10 & 30 & 24 & $5: 1$ & 88.0 & [189] \\
\hline $\begin{array}{l}\text { Palm oil fatty } \\
\text { acid distillate } \\
\text { (PFAD) }\end{array}$ & $\begin{array}{l}\text { Candida } \\
\text { antarctica }\end{array}$ & Novozyme 435 & 1 & 60 & 2.5 & $3: 1$ & 93.0 & [190] \\
\hline Tung oil & Rhizopus oryzae & $\begin{array}{l}\text { Chimeric } \\
\text { lipase }\end{array}$ & 13 & 40 & 48 & 3.88 & 91.9 & [191] \\
\hline Jatropha oil & $\begin{array}{l}\text { Enterobacter } \\
\text { aerogenes }\end{array}$ & Lipase & $-*$ & 55 & 48 & $4: 1$ & 94.0 & [192] \\
\hline Waste tallow & $\begin{array}{l}\text { Candida } \\
\text { antarctica }\end{array}$ & $\begin{array}{l}\text { Lipase B } \\
\text { (CALB) } \\
\text { Candida } \\
\text { antarctica } \\
\text { lipase B }\end{array}$ & 1.25 & 50 & 24 & $30: 1$ & 99.0 & [193] \\
\hline $\begin{array}{l}\text { Nanochloropsis } \\
\text { oculata } \\
\text { microalga }\end{array}$ & Bacillus sp. S23 & Lipase & 1.5 & 35 & 60 & $12: 1$ & 95.7 & [194] \\
\hline Beef tallow & $\begin{array}{l}\text { Burkholderia } \\
\text { cepacia }\end{array}$ & $\begin{array}{c}\text { Immobilized } \\
\text { lipase }\end{array}$ & 20 & 50 & 48 & $12: 1$ & 89.7 & [195] \\
\hline Animal fat & $\begin{array}{l}\text { Candida } \\
\text { antarctica }\end{array}$ & $\begin{array}{c}\text { Immobilized } \\
\text { lipase }\end{array}$ & 10 & 40 & 6 & $50: 6$ & 79.0 & [196] \\
\hline Lard & Candida sp. & Lipase & 20 & 40 & 30 & $3: 1$ & 87.4 & [197] \\
\hline Lard & $\begin{array}{l}\text { Candida } \\
\text { antarctica }\end{array}$ & Lipase & 10 & 30 & 72 & $1: 1$ & 74.0 & [198] \\
\hline Lard & $\begin{array}{l}\text { Candida } \\
\text { antarctica }\end{array}$ & Lipase & $2-6$ & 50 & 20 & $5: 1$ & 97.2 & [199] \\
\hline $\begin{array}{c}\text { Used } \\
\text { cottonseed oil }\end{array}$ & $\begin{array}{l}\text { Pseudomonas } \\
\text { sp. }\end{array}$ & Lipase & 30 & 37 & 48 & $6: 1$ & 70.0 & [200] \\
\hline Palm oil & $\begin{array}{c}\text { Rhodotorula } \\
\text { mucilagenosa } \mathrm{P} \\
11189\end{array}$ & Lipase & 0.5 & 30 & 72 & $3: 1$ & 51.3 & [201] \\
\hline Palm oil & $\begin{array}{l}\text { Aspergillus } \\
\text { niger }\end{array}$ & Lipase & $2-3$ & 25 & 72 & $3: 1$ & 87.0 & [202] \\
\hline Palm oil & $\begin{array}{l}\text { Aspergillus } \\
\text { niger }\end{array}$ & Lipase & $2-3$ & 40 & 72 & $3: 1$ & 69.0 & [202] \\
\hline $\begin{array}{l}\text { Used cooking } \\
\text { oil }\end{array}$ & $\begin{array}{l}\text { Rhizopus oryzae } \\
\text { PTCC } 5174\end{array}$ & Lipase & 15.5 & 35 & 72 & $3: 1$ & 98.0 & [203] \\
\hline Soybean oil & Rhizopus oryzae & Lipase & 5 & 35 & 72 & $3: 1$ & 89-92 & [204] \\
\hline
\end{tabular}

* $50 \mathrm{U}$ of immobilized lipase/g. 


\section{Lipase and Biodiesel Markets}

\subsection{Lipase Market}

According to the Fior Market survey, the global lipase market is projected to register a CAGR of $8.8 \%$ during the forecast period 2020-2025 and reach USD 961.85 million by 2028 [205]. Due to the diverse origin of lipases, their properties, and their abilities to catalyze different biotechnical reactions, the market for lipases is expanding beyond the food and biofuels industry to include animal feed, pharmaceutical, detergent, cosmetic, pulp and paper, leather, and textile industries [206]. The use of lipase in biology and electronics, biosensors, and nanotechnology is also on the rise. The global lipase market is segmented into animal lipases and microbial lipases. In 2020, the microbial and animal feed lipases segments held the largest market share of $61.64 \%$ and $26.6 \%$, respectively. The key players in the global lipase market are Amano Enzymes Inc. (Elgin, IL, USA), Advanced Enzymes (Thane, Maharashtra, India), Clerici-Sacco Group (Cadorago, Como, Italy), Chr. Hansen Holdings A/S (Boege Alle, Hoersholm, Denmark), Enzyme Development Corporation (New York, NY, USA), E. I. Du Pont De Nemours (Wilmington, DE, USA), Genencor (Rochester, NY, USA), Novozymes A/S (Franklinton, NC, USA), Koninklijke DSM N.V. (Heerlen, Limburg, The Netherlands), and Renco New Zealand (Eltham, Taranaki, New Zealand) [207]. The major limitations for the wider, large-scale use of lipases have been their high cost and the lack of transparency in the laws and regulations related to lipase patents around the world.

\subsection{Biodiesel Market}

In 2016, the size of the biodiesel market was USD 34.1 billion and should reach USD 41.2 billion by 2021 at a 3.8\% CAGR. However, over the period 2021-2028, the global biodiesel market is forecasted to grow at a higher CAGR of $5.25 \%$. The biodiesel demand is expected to exceed 41.4 billion liters by 2025 [208]. The biodiesel market is driven by the increasing demand for environmentally safe fuels that reduce GHG emissions. Other major growthinducing factors are the thriving automotive industry, the surging prices of fossil fuels, the recent advancements and emergence of the third-generation biodiesel from algae, and the implementation of favorable government policies to promote biodiesel usage [209]. Chemical biodiesel is manufactured globally by several major producers, such as Archer Daniels Midland Company (Chicago, IL, USA), Wilmar International Limited (Chinatown, Singapore), Bunge Limited (Chesterfield, MO, USA), Neste Corporation (Espoo, Finland), Renewable Energy Group Inc. (Ames, IA, USA), Louis Dreyfus Company (Rotterdam, The Netherlands), Cargill Inc. (Wayzata, MN, USA), BIOX Corporation (Hamilton, ON, Canada), Munzer Bioindustrie (Vienna, Austria), and Emami Group (Kolkata, West Bengal, India).

Although the enzymatic routes for biodiesel production have been intensively investigated, the large-scale production of enzymatic biodiesel is currently limited (Table 6). The U.S. has several commercial-scale production facilities; however, the largest single plant (Aemetis Biorefinery, Inc., Cupertino, CA, USA) for enzymatic biodiesel production of 50 million gallons per year has been built in India. International collaborative efforts and strategic partnerships between biodiesel manufacturers and lipase-producing companies may accelerate the progress towards the development of commercially viable technologies for enzymatic biodiesel. 
Table 6. Commercial production of enzymatic biodiesel: lipase source, feedstock, and annual production of biodiesel.

\begin{tabular}{|c|c|c|c|c|c|}
\hline Country & Company Name & Lipase Source & Main Feedstock & Annual Production & Reference \\
\hline USA & Viesel Fuel LLC & $\begin{array}{l}\text { Eversa Transform }{ }^{\circledR} \\
\quad \text { from A. oryzae }\end{array}$ & $\begin{array}{l}\text { Waste cooking oil, } \\
\text { brown grease }\end{array}$ & 11 million gallons & [210] \\
\hline USA & $\begin{array}{c}\text { SRS International } \\
\text { Co. }\end{array}$ & Immobilized lipase & Used restaurant oil & 5 million gallons & [211] \\
\hline USA & Buster Biofuels & $\begin{array}{c}\text { Callera }^{\circledR} \text { Trans L } \\
\text { lipase from } \\
\text { Thermomyces } \\
\text { lanuginosus }\end{array}$ & Brown grease, fish oil & 5 million gallons & [212] \\
\hline USA & Blue Sun Energy & $\begin{array}{c}\text { Callera }{ }^{\circledR} \text { Trans L } \\
\text { lipase from } \\
\text { Thermomyces } \\
\text { lanuginosus }\end{array}$ & $\begin{array}{l}\text { Used cooking oil, palm } \\
\text { fatty acid distillate, } \\
\text { corn oil }\end{array}$ & 30 million gallons & [213] \\
\hline Israel & TransBiodiesel Ltd. & TransZyme A & $\begin{array}{l}\text { Used cooking oil, } \\
\text { animal fat, acid oil, } \\
\text { brown grease }\end{array}$ & 50,000 tons & [214] \\
\hline Israel & EnzymeCore & TransZyme A & $\begin{array}{l}\text { Low-cost oils and fats } \\
\text { with high free fatty } \\
\text { acid and polar lipid } \\
\text { content }\end{array}$ & 1500 tons & [215] \\
\hline South Korea & M-Energy & TransZyme A & $\begin{array}{c}\text { Brown grease } \\
\text { extracted from grease } \\
\text { trap, fat, oil, grease }\end{array}$ & 30,000 tons & [216] \\
\hline India & $\begin{array}{c}\text { Aemetis } \\
\text { Biorefinery, Inc. }\end{array}$ & - & $\begin{array}{l}\text { Brown grease, low } \\
\text { grade used cooking } \\
\text { oils, palm fatty acid } \\
\text { distillate and other } \\
\text { plant oil waste } \\
\text { feedstocks }\end{array}$ & 50 million gallons & [217] \\
\hline China & $\begin{array}{l}\text { Lvming and } \\
\text { Environmental } \\
\text { Protection } \\
\text { Technology Co. } \\
\text { Ltd. }\end{array}$ & $\begin{array}{c}\text { Candida sp. } 99-125 \\
\text { lipase }\end{array}$ & Waste cooking oil & 10,000 tons & [218] \\
\hline China & $\begin{array}{c}\text { Hunan Rivers } \\
\text { Bioengineering Co. } \\
\text { Ltd. }\end{array}$ & $\begin{array}{l}\text { Immobilized } \\
\text { Novozym } 435^{\circledR} \\
\text { (lipase B from } \\
\text { Candida antarctica) }\end{array}$ & $\begin{array}{c}\text { Beef tallow, soybean } \\
\text { oil }\end{array}$ & 20,000 tons & [218] \\
\hline Brazil & Olfar & $\begin{array}{c}\text { Immobilized } \\
\text { Callera }{ }^{\circledR} \text { Trans L } \\
\text { lipase from } \\
\text { Thermomyces } \\
\text { lanuginosus }\end{array}$ & $\begin{array}{l}\text { Recovered vegetable } \\
\text { oil, animal fat, } \\
\text { soybean oil }\end{array}$ & 378 million liters & [219] \\
\hline
\end{tabular}

As evident from Table 6, the predominant raw materials for the commercial production of enzymatic biodiesel are waste cooking oil and brown grease, both second-generation feedstock. Waste cooking/frying oils have high FFA content (typically 20-60\% w/w) as heat and water are known to accelerate the hydrolysis of TAG to FFA. On the other hand, brown grease is categorized as grease that contains above $15 \% w / w$ FFA. The alkalicatalyzed transesterification reaction of waste cooking oil and brown grease results in soap formation, which consumes the alkali catalyst, creates difficulties in the downstream recovery of biodiesel and diminishes the biodiesel yield. Pretreatment with sulfuric acid in the presence of methanol is required to esterify the FFA and reduce their content to 
below $1 \%(w / w)$ before the alkaline transesterification of TAG to FAME can proceed. The two-step acid-base process adds to the production cost and is considered as one of the major drawbacks of chemical biodiesel [16]. Lipases can trans/esterify both TAG and FFA in one step, hence their use on a large scale. Table 6 also suggests that the commercial process makes use of commercial lipases as the preferred biocatalyst in both free (liquid) and immobilized (solid) states.

\section{Concluding Remarks}

Biodiesel is a renewable, biodegradable, non-flammable, non-toxic biofuel that has the potential to minimize the use of petrodiesel and strengthen energy security and socioeconomic development while reducing the environmental impact. To reach its full potential as the energy source of choice, several techno-economic and social challenges need to be overcome.

The main challenge in enzymatic biodiesel production is its high cost. Lipases are still expensive, and the enzyme-catalyzed process requires more time to complete than the alkali-catalyzed process. If not optimized, lipases may be inhibited by methanol, which results in diminished biodiesel yields. The cost-efficiency of biodiesel can be improved using immobilized enzymes that are recovered and recycled. As the glycerol co-product in biodiesel production remains underutilized, the development of viable technologies for glycerol conversion to value-added products will further strengthen the production economics of biodiesel. The ability of lipase to catalyze biodiesel production from lowcost feedstock with a high free fatty acid content, such as waste cooking oil, grease, and tallow, can also lower the cost of enzymatic biodiesel. Furthermore, the discovery and engineering of new and robust lipases with high activity, thermostability, and resistance to inhibition will accelerate the progress towards the establishment of a cost-effective enzymatic process [23]. Another challenge is the poor cold flow properties of biodiesel in terms of cloud point, pour point, and cold filter plugging point [83,220-223]. The presence of higher amounts of FAME in biodiesel than petrodiesel further aggravates this problem. In addition, the water content of oily feedstocks may need adjustment to ensure optimal transesterification conditions for lipase catalysis. A further drawback is the low oxidation stability of biodiesel (both enzyme- and alkali-catalyzed biodiesel) that is caused by the presence of polyunsaturated FAME [224]. In addition, the viscosity of biodiesel is 11-17 times higher than petrodiesel, which can make pumping, combustion, and atomization more difficult [225]. Biodiesel combustion may lead to sludge buildup in the injectors and engine heads, and compression ignition engines can wear excessively [226]. The use of fuel additives can improve the oxidation stability, engine, and storage life of biodiesel, and the performance of biodiesel-diesel blends in cold climates $[227,228]$. However, there is a paucity of studies on the effects of antioxidants on the lubricity of biodiesel while patents regarding methods for improving the cold flow properties of biodiesel remain scarce $[229,230]$. For this reason, the biodiesel's physical-chemical properties need to be refined to increase compatibility with compression ignition engines.

A major disadvantage of the first-generation biodiesel is that it is produced from edible oils derived from food crops, hence the concerns about increases in food prices in the global market and the ongoing food vs. fuel debate. While the second-generation biodiesel avoids the use of food crops, it still requires the cultivation of non-edible oil plants, which may lead to competition with food crops for arable land [231]. In addition to the above challenges, the expansion of the biodiesel industry may have direct or indirect negative environmental impacts. For example, to maintain a sufficient feedstock supply, more land may be required to cultivate oil crops, which could lead to deforestation. Rainforests are the largest carbon sinks in the world, and their removal will inevitably release enormous amounts of carbon into the atmosphere. Furthermore, as water is extensively used for cooking, drinking, and irrigation, water-intensive biodiesel production can considerably strain water resources and increase pressures on water supplies [232]. 
Farmers, researchers, feedstock providers, transporter, investors, and youth are expected to benefit from the emerging biodiesel market. Many policymakers favor biodiesel for its potential benefits, including increased domestic energy stability, lower GHG emissions, economic growth, and job creation, especially in rural areas. Subsidies, grants, campaigns, and biodiesel mandates in national policies can be implemented to facilitate the development of first-generation biodiesel. A study of the socio-economic status in Thailand related to the biodiesel market reported that more workers are needed for the biodiesel than the petrodiesel industry, which will create more employment by the end of 2022 [233]. Likewise, India currently generates around 70 billion liters of wastewater per day, and opportunities exist to upgrade the existing sewage treatment plants for simultaneous production of biogas and biodiesel. This may add an additional 700,000 jobs and allow the production of around 350 million liters of biodiesel. Various national and international organizations currently collaborate with the objective to demonstrate realworld opportunities of innovative biodiesel technology for the production and usage of high-mileage $100 \%$ biodiesel (B100) in vehicle applications. A variety of cooperation initiatives engage sustainable diesel supporters around the world to promote biodiesel and its awareness at the regional, national, and international levels. These include National Biodiesel Board (https:/ /www.biodiesel.org, accessed on 20 August 2021), European Biodiesel Board (https:/ / ebb-eu.org, accessed on 20 August 2021), Biodiesel Ambassadors (https:/ / www.nbb.org/join-us/partnership-programs/biodiesel-ambassadors, accessed on 20 August 2021), Biodiesel Alliance (https://www.greenamerica.org/fuels-future/ sustainable-biodiesel-alliance, accessed on 20 August 2021), Next Generation Scientists for Biodiesel, (https:/ /biodieselsustainability.org/ngsb/, accessed on 20 August 2021), and others.

Author Contributions: Conceptualization, supervision, resources, writing-original draft preparation, writing — review and editing, V.Z.; writing — original draft preparation, R.P.; writing — review and editing, B.B.; conceptualization, supervision, writing-review, editing, preparation of final draft, submission, L.C. All authors have read and agreed to the published version of the manuscript.

Funding: This research received no external funding.

Institutional Review Board Statement: Not applicable.

Informed Consent Statement: Not applicable.

Conflicts of Interest: The authors declare no conflict of interest.

\section{References}

1. Fukuda, H.; Kondo, A.; Noda, H. Biodiesel fuel production by transesterification of oils. J. Biosci. Bioeng. 2001, 92, 405-416. [CrossRef]

2. Abbaszaadeh, A.; Ghobadian, B.; Omidkhah, M.R.; Najafi, G. Current biodiesel production technologies: A comparative review. Energy Convers. Manag. 2012, 63, 138-148. [CrossRef]

3. De Oliveira, F.C.; Coelho, S.T. History, evolution, and environmental impact of biodiesel in Brazil: A review. Renew. Sustain. Energy Rev. 2017, 75, 168-179. [CrossRef]

4. Alberts, G.; Ayuso, M.; Bauen, A.; Boshell, F.; Chudziak, C.; Gebauer; Peer, J.; German, L.; Kaltschmitt; Martin; et al. Inovation Outlook: Advanced Liquid Biofuels; International Renewable Energy Agency (IRENA): New York, NY, USA, 2016; ISBN 978-9295111-52-3.

5. Jain, S.K.; Kumar, S.; Chaube, A. Technical sustainability of biodiesel and its blends with diesel in ci engines: A review. Int. J. Chem. Eng. Appl. 2011, 2, 101-109.

6. Hasan, M.M.; Rahman, M.M. Performance and emission characteristics of biodiesel-diesel blend and environmental and economic impacts of biodiesel production: A review. Renew. Sustain. Energy Rev. 2017, 74, 938-948. [CrossRef]

7. MIT. MIT Energy Initiative Symposium on Prospects for Bi-Fuel and Flex-Fuel Light-Duty Vehicles; Massachusetts Institute of Technology: Cambridge, MA, USA, 2012.

8. Gebremariam, S.N.; Marchetti, J.M. Economics of biodiesel production: Review. Energy Convers. Manag. 2018, 168, 74-84. [CrossRef]

9. Christopher, L.P.; Kumar, H. Clean and Sustainable Biodiesel Production. In Handbook of Clean Energy Systems; Yan, J., Ed.; John Wiley \& Sons, Ltd.: Chichester, UK, 2015; pp. 1-16. 
10. Lim, S.; Teong, L.K. Recent trends, opportunities and challenges of biodiesel in Malaysia: An overview. Renew. Sustain. Energy Rev. 2010, 14, 938-954. [CrossRef]

11. Mofijur, M.; Mahlia, T.M.I.; Silitonga, A.S.; Ong, H.C.; Silakhori, M.; Hasan, M.H.; Putra, N.; Ashrafur Rahman, S.M. Phase change materials (PCM) for solar energy usages and storage: An overview. Energies 2019, 12, 3167. [CrossRef]

12. Mujtaba, M.A.; Muk Cho, H.; Masjuki, H.H.; Kalam, M.A.; Ong, H.C.; Gul, M.; Harith, M.H.; Yusoff, M.N.A.M. Critical review on sesame seed oil and its methyl ester on cold flow and oxidation stability. Energy Rep. 2020, 6, 40-54. [CrossRef]

13. Ishola, F.; Adelekan, D.; Mamudu, A.; Abodunrin, T.; Aworinde, A.; Olatunji, O.; Akinlabi, S. Biodiesel production from palm olein: A sustainable bioresource for Nigeria. Heliyon 2020, 6, e03725. [CrossRef]

14. Mitrović, P.M.; Stamenković, O.S.; Banković-Ilić, I.; Djalović, I.G.; Nježić, Z.B.; Farooq, M.; Siddique, K.H.M.; Veljković, V.B. White mustard (Sinapis alba L.) oil in biodiesel production: A review. Front. Plant Sci. 2020, 11, 299. [CrossRef]

15. Yusoff, M.N.A.M.; Zulkifli, N.W.M.; Sukiman, N.L.; Chyuan, O.H.; Hassan, M.H.; Hasnul, M.H.; Zulkifli, M.S.A.; Abbas, M.M.; Zakaria, M.Z. Sustainability of Palm Biodiesel in Transportation: A Review on Biofuel Standard, Policy and International Collaboration between Malaysia and Colombia. Bioenergy Res. 2021, 14, 43-60. [CrossRef] [PubMed]

16. Su, J.J.; Chou, Y.C. Biodiesel production by acid methanolysis of slaughterhouse sludge cake. Animals 2019, 9, 1029. [CrossRef] [PubMed]

17. Okoro, O.V.; Sun, Z.; Birch, J. Meat processing waste as a potential feedstock for biochemicals and biofuels-A review of possible conversion technologies. J. Clean. Prod. 2017, 142, 1583-1608. [CrossRef]

18. Chua, S.Y.; Periasamy, L.A.; Goh, C.M.H.; Tan, Y.H.; Mubarak, N.M.; Kansedo, J.; Khalid, M.; Walvekar, R.; Abdullah, E.C. Biodiesel synthesis using natural solid catalyst derived from biomass waste-A review. J. Ind. Eng. Chem. 2020, 81, 41-60. [CrossRef]

19. Demirbas, A. Production of biodiesel fuels from linseed oil using methanol and ethanol in non-catalytic SCF conditions. Biomass Bioenergy 2009, 33, 113-118. [CrossRef]

20. Dabi, M.; Saha, U.K. Application potential of vegetable oils as alternative to diesel fuels in compression ignition engines: A review. J. Energy Inst. 2019, 92, 1710-1726. [CrossRef]

21. Ramli, A.; Farooq, M.; Naeem, A.; Khan, S.; Hummayun, M.; Iqbal, A.; Ahmed, S.; Shah, L.A. Bifunctional Heterogeneous Catalysts for Biodiesel Production using Low Cost Feedstocks: A Future Perspective. In Frontiers in Bioenergy and Biofuels; Jacob-Lopes, E., Zepka, L.Q., Eds.; IntechOpen Limited: London, UK, 2017; pp. 285-308.

22. Shah, S.; Sharma, S.; Gupta, M.N. Enzymatic transesterification for biodiesel production. Indian J. Biochem. Biophys. 2003, 40, 392-399.

23. Christopher, L.P.; Kumar, H.; Zambare, V.P. Enzymatic biodiesel: Challenges and opportunities. Appl. Energy 2014, 119, 497-520. [CrossRef]

24. Ondul, E.; Dizge, N.; Keskinler, B.; Albayrak, N. Biocatalytic Production of Biodiesel from Vegetable Oils. In Biofuels-Status and Perspective; IntechOpen Limited: London, UK, 2015.

25. Karmakar, B.; Halder, G. Progress and future of biodiesel synthesis: Advancements in oil extraction and conversion technologies. Energy Convers. Manag. 2019, 182, 307-339. [CrossRef]

26. Chilakamarry, C.R.; Mimi Sakinah, A.M.; Zularisam, A.W.; Pandey, A.; Vo, D.-V.N. Technological perspectives for utilisation of waste glycerol for the production of biofuels: A review. Environ. Technol. Innov. 2021, 24, 101902. [CrossRef]

27. Gautam, R.; Vinu, R. Reaction engineering and kinetics of algae conversion to biofuels and chemicals via pyrolysis and hydrothermal liquefaction. React. Chem. Eng. 2020, 5, 1320-1373. [CrossRef]

28. Hama, S.; Kondo, A. Enzymatic biodiesel production: An overview of potential feedstocks and process development. Bioresour. Technol. 2013, 135, 386-395. [CrossRef] [PubMed]

29. Chozhavendhan, S.; Vijay Pradhap Singh, M.; Fransila, B.; Praveen Kumar, R.; Karthiga Devi, G. A review on influencing parameters of biodiesel production and purification processes. Curr. Res. Green Sustain. Chem. 2020, 1-2, 1-6. [CrossRef]

30. Zhang, H.; Han, L.; Dong, H. An insight to pretreatment, enzyme adsorption and enzymatic hydrolysis of lignocellulosic biomass: Experimental and modeling studies. Renew. Sustain. Energy Rev. 2021, 140, 110758. [CrossRef]

31. Moser, B.R. Biodiesel production, properties, and feedstocks. In Vitro Cell. Dev. Biol. Plant 2009, 45, 229-266. [CrossRef]

32. Hill, J.; Nelson, E.; Tilman, D.; Polasky, S.; Tiffany, D. Environmental, economic, and energetic costs and benefits of biodiesel and ethanol biofuels. Proc. Natl. Acad. Sci. USA 2006, 103, 11206-11210. [CrossRef]

33. Varela, V.J.; Burgués, C.; Rösch, C. Acceptability of genetically engineered algae biofuels in Europe: Opinions of experts and stakeholders. Biotechnol. Biofuels 2020, 13, 92. [CrossRef]

34. Yu, A.; Zhao, Y.; Li, J.; Li, S.; Pang, Y.; Zhao, Y.; Zhang, C.; Xiao, D. Sustainable production of FAEE biodiesel using the oleaginous yeast Yarrowia lipolytica. Microbiologyopen 2020, 9, e1051. [CrossRef]

35. Rahman, Z.; Sung, B.H.; Nawab, J.; Siddiqui, M.F.; Ali, A.; Geraldi, A.; Kim, S.C. Enhanced production of fatty acid ethyl ester with engineered fabhdg operon in escherichia coli. Microorganisms 2019, 7, 552. [CrossRef]

36. Wahlen, B.D.; Morgan, M.R.; McCurdy, A.T.; Willis, R.M.; Morgan, M.D.; Dye, D.J.; Bugbee, B.; Wood, B.D.; Seefeldt, L.C. Biodiesel from microalgae, yeast, and bacteria: Engine performance and exhaust emissions. Energy Fuels 2013, 27, 220-228. [CrossRef]

37. De Paola, M.G.; Mazza, I.; Paletta, R.; Lopresto, C.G.; Calabrò, V. Small-Scale Biodiesel Production Plants-An Overview. Energies 2021, 14, 1901. [CrossRef] 
38. Rao, K.S.; Ramakrishna, A. Cost Estimation Analysis of Biodiesel Production from Waste Chicken Fat. Int. J. Appl. Eng. Res. 2015, 10, 8863-8870.

39. Joelsson, E.; Erdei, B.; Galbe, M.; Wallberg, O. Techno-economic evaluation of integrated first- and second-generation ethanol production from grain and straw. Biotechnol. Biofuels 2016, 9, 1. [CrossRef]

40. Behera, S.; Singh, R.; Arora, R.; Sharma, N.K.; Shukla, M.; Kumar, S. Scope of Algae as Third Generation Biofuels. Front. Bioeng. Biotechnol. 2015, 2, 90. [CrossRef]

41. Vingering, N.; Oseredczuk, M.; Du Chaffaut, L.; Ireland, J.; Ledoux, M. Fatty acid composition of commercial vegetable oils from the French market analysed using a long highly polar column. OCL-Ol. Corps Gras Lipides 2010, 17, 185-192. [CrossRef]

42. Serin, H.; Ozcanli, M.; Gokce, M.K.; Tuccar, G. Biodiesel production from tea seed (camellia sinensis) oil and its blends with diesel fuel. Int. J. Green Energy 2013, 10, 370-377. [CrossRef]

43. Deeba, F.; Patel, A.; Arora, N.; Pruthi, V.; Pruthi, P.A.; Negi, Y.S. Amaranth seeds (Amaranthus palmeri L.) as novel feedstock for biodiesel production by oleaginous yeast. Environ. Sci. Pollut. Res. 2018, 25, 353-362. [CrossRef] [PubMed]

44. Anwar, F.; Rashid, U.; Ashraf, M.; Nadeem, M. Okra (Hibiscus esculentus) seed oil for biodiesel production. Appl. Energy 2010, 87, 779-785. [CrossRef]

45. Hellier, P.; Ladommatos, N.; Yusaf, T. The influence of straight vegetable oil fatty acid composition on compression ignition combustion and emissions. Fuel 2015, 143, 131-143. [CrossRef]

46. Awogbemi, O.; Onuh, E.I.; Inambao, F.L. Comparative study of properties and fatty acid composition of some neat vegetable oils and waste cooking oils. Int. J. Low-Carbon Technol. 2019, 14, 417-425. [CrossRef]

47. Verma, R.; Sharma, D.K.; Bisen, P.S. Determination of Free Fatty Acid Composition in Jatropha Crude Oil and Suitability as Biodiesel Feedstock. Curr. Altern. Energy 2019, 3, 59-64. [CrossRef]

48. Rahangdale, C.P.; Koshta, L.D.; Patle, N.K. Provenance Variation for Oil Content and Fatty Acid Composition in Seed of Pongamia pinnata (L.) Pierre. Int. J. Proj. Manag. 2014, 4, 1-9.

49. Kulkarni, P.S.; Ramesh, M.R.; Sharanappa, G. Mahua (Madhuca indica) as a Source of Biodiesel in India. Int. J. Sci. Eng. Res. 2013, 4, 2319-2329.

50. Omohu, J.O. Physicochemical properties and fatty acid composition of castor bean Ricinus communis L. seed oil. Eur. J. Biophys. 2017, 5, 62. [CrossRef]

51. Sandanasamy, J.D. Fatty acid composition and antibacterial activity of neem (Azadirachta indica) seed oil. Open Conf. Proc. J. 2014, 4, 43-48. [CrossRef]

52. Al-Rashed, S.A.; Ibrahim, M.M.; Hatata, M.M.A.; El-Gaaly, G.A. Biodiesel production and antioxidant capability from seeds of Salicornia begelovii collected from Al Jubail, Eastern province, Saudi Arabia. Pak. J. Bot. 2016, 48, 2527-2533.

53. Nayak, S.K.; Mishra, P.C. Application of Nagchampa biodiesel and rice husk gas as fuel. Energy Sources Part A Recovery Util. Environ. Eff. 2016, 38, 2024-2030. [CrossRef]

54. Mohd-Setapar, H.S.; Nian-Yian, L.; Kamarudin, N.W.W.; Idham, Z.; Norfahana, A.-T. Omega-3 emulsion of Rubber (Hevea brasiliensis) seed oil. Agric. Sci. 2013, 4, 84-89. [CrossRef]

55. Mohammad, M. Evaluation of Chemical Compositions of Tobacco (Nicotiana tabacum L) Genotypes Seeds. Annu. Res. Rev. Biol. 2014, 4, 1480-1489. [CrossRef]

56. Moser, B.R.; Knothe, G.; Cermak, S.C. Biodiesel from meadowfoam (Limnanthes alba L.) seed oil: Oxidative stability and unusual fatty acid composition. Energy Environ. Sci. 2010, 3, 318-327. [CrossRef]

57. Banani, R.; Youssef, S.; Bezzarga, M.; Abderrabba, M. Waste frying oil with high levels of free fatty acids as one of the prominent sources of biodiesel production. J. Mater. Environ. Sci. 2015, 6, 1178-1185.

58. Liu, Y.; Liu, H.; Huang, L.; Gui, S.; Zheng, D.; Jia, L.; Fu, Y.; Lu, F. Improvement in thermostability of an alkaline lipase i from: Penicillium cyclopium by directed evolution. RSC Adv. 2017, 7, 38538-38548. [CrossRef]

59. Toldrá, F.; Rubio, M.A.; Navarro, J.L.; Cabrerizo, L. Quality aspects of pork meat and its nutritional impact. Adv. Exp. Med. Biol. 2004, 542, 25-31. [CrossRef] [PubMed]

60. Realini, C.E.; Duckett, S.K.; Brito, G.W.; Dalla Rizza, M.; De Mattos, D. Effect of pasture vs. concentrate feeding with or without antioxidants on carcass characteristics, fatty acid composition, and quality of Uruguayan beef. Meat Sci. 2004, 66, 567-577. [CrossRef]

61. Encinar, J.M.; Sánchez, N.; Martínez, G.; García, L. Study of biodiesel production from animal fats with high free fatty acid content. Bioresour. Technol. 2011, 102, 10907-10914. [CrossRef] [PubMed]

62. Zduńczyk, Z.; Gruzauskas, R.; Semaskaite, A.; Juskiewicz, J.; Raceviciute-Stupeliene, A.; Wroblewska, M. Fatty acid profile of breast muscle of broiler chickens fed diets with different levels of selenium and vitamin E. Arch. Geflugelkd. 2011, 75, $264-267$.

63. Gandure, J.; Ketlogetswe, C.; Jonas, M. Production, Composition and Fuel Properties of Tallow Biodiesel: A Case of Botswana. Energy Power Eng. 2017, 9, 355-365. [CrossRef]

64. Cunha, A.; Feddern, V.; De Prá, M.C.; Higarashi, M.M.; de Abreu, P.G.; Coldebella, A. Synthesis and characterization of ethylic biodiesel from animal fat wastes. Fuel 2013, 105, 228-234. [CrossRef]

65. Mata, T.M.; Martins, A.A.; Caetano, N.S. Microalgae for biodiesel production and other applications: A review. Renew. Sustain. Energy Rev. 2010, 14, 217-232. [CrossRef] 
66. Du, Z.-Y.; Alvaro, J.; Hyden, B.; Zienkiewicz, K.; Benning, N.; Zienkiewicz, A.; Bonito, G.; Benning, C. Enhancing oil production and harvest by combining the marine alga Nannochloropsis oceanica and the oleaginous fungus Mortierella elongata. Biotechnol. Biofuels 2018, 11, 174. [CrossRef]

67. Xue, J.; Niu, Y.-F.; Huang, T.; Yang, W.-D.; Liu, J.-S.; Li, H.-Y. Genetic improvement of the microalga Phaeodactylum tricornutum for boosting neutral lipid accumulation. Metab. Eng. 2015, 27, 1-9. [CrossRef]

68. Zulu, N.N.; Popko, J.; Zienkiewicz, K.; Tarazona, P.; Herrfurth, C.; Feussner, I. Heterologous co-expression of a yeast diacylglycerol acyltransferase (ScDGA1) and a plant oleosin (AtOLEO3) as an efficient tool for enhancing triacylglycerol accumulation in the marine diatom Phaeodactylum tricornutum. Biotechnol. Biofuels 2017, 10, 187. [CrossRef]

69. Shokravi, Z.; Shokravi, H.; Aziz, M.A.; Shokravi, H. The Fourth-Generation Biofuel: A Systematic Review on Nearly Two Decades of Research from 2008 to 2019. In Fossil Free Fuels; CRC Press: Boca Raton, FL, USA, 2019; pp. 213-251.

70. Basumatary, S. Yellow oleander (Thevetia peruviana) seed oil biodiesel as an alternative and renewable fuel for diesel engines: A review. Int. J. ChemTech Res. 2014, 7, 2823-2840.

71. Tupa, R.; Silalahi, F.; Simatupang, T.M.; Siallagan, M.P. Biodiesel produced from palm oil in Indonesia: Current status and opportunities. AIMS Energy 2020, 8, 81-101. [CrossRef]

72. Thushari, I.; Babel, S.; Samart, C. Biodiesel production in an autoclave reactor using waste palm oil and coconut coir husk derived catalyst. Renew. Energy 2019, 134, 125-134. [CrossRef]

73. Kumar Naik, M.; Charan Meher, L.; Kumar Dalai, A.; Narayan Naik, S. Biodiesel Production Using Karanja (Pongamia pinnata) and Jatropha (Jatropha curcas) Seed Oil. In Handbook of Plant-Based Biofuels; CRC Press: Boca Raton, FL, USA, 2008 ; pp. 255-266.

74. Sahu, G.; Das, L.M.; Sharma, B.K.; Naik, S.N. Pilot plant study on biodiesel production from Karanja and Jatropha oils. Asia-Pac. J. Chem. Eng. 2011, 6, 38-43. [CrossRef]

75. Chhetri, A.; Tango, M.; Budge, S.; Watts, K.; Islam, M. Non-Edible Plant Oils as New Sources for Biodiesel Production. Int. J. Mol. Sci. 2008, 9, 169-180. [CrossRef]

76. Jena, P.C.; Raheman, H.; Prasanna Kumar, G.V.; Machavaram, R. Biodiesel production from mixture of mahua and simarouba oils with high free fatty acids. Biomass Bioenergy 2010, 34, 1108-1116. [CrossRef]

77. Alamu, O.J.; Dehinbo, O.; Sulaiman, A.M. Production and testing of coconut oil biodiesel fuel and its blend. Leonardo J. Sci. 2010, $16,95-104$.

78. Mekhilef, S.; Siga, S.; Saidur, R. A review on palm oil biodiesel as a source of renewable fuel. Renew. Sustain. Energy Rev. 2011, 15, 1937-1949. [CrossRef]

79. Mariano, G.C.; Lopes, T.J.; Dias, R.; Bastos Quadri, M.; Bolzan, A. Production of Biodiesel with Seed Soybean and Supercritical Ethanol. J. Sustain. Bioenergy Syst. 2014, 4, 128-135. [CrossRef]

80. Mansourpoor, M. Optimization of biodiesel production from Sunflower Oil Using Response Surface Methodology. J. Chem. Eng. Process Technol. 2012, 03, 3-7. [CrossRef]

81. Ruan, R.; Zhang, Y.; Chen, P.; Liu, S.; Fan, L.; Zhou, N.; Ding, K.; Peng, P.; Addy, M.; Cheng, Y.; et al. Biofuels: Introduction. In Biofuels: Alternative Feedstocks and Conversion Processes for the Production of Liquid and Gaseous Biofuels; Elsevier: Amsterdam, The Netherlands, 2019; pp. 3-43.

82. Groves, C.; Shankar, M.; Thomas, P.J. Second-generation biofuels: Exploring imaginaries via deliberative workshops with farmers. J. Responsible Innovat. 2018, 5, 149-169. [CrossRef]

83. Singh, D.; Sharma, D.; Soni, S.L.; Inda, C.S.; Sharma, S.; Sharma, P.K.; Jhalani, A. A Comprehensive Review on 1st-Generation Biodiesel Feedstock Palm Oil: Production, Engine Performance, and Exhaust Emissions. Bioenergy Res. 2020, 14, 1-22. [CrossRef]

84. Foidl, N.; Foidl, G.; Sanchez, M.; Mittelbach, M.; Hackel, S. Jatropha curcas L. as a source for the production of biofuel in Nicaragua. Bioresour. Technol. 1996, 58, 77-82. [CrossRef]

85. Siler-Marinkovic, S.; Tomasevic, A. Transesterification of sunflower oil in situ. Fuel 1998, 77, 1389-1391. [CrossRef]

86. Ghadge, S.V.; Raheman, H. Process optimization for biodiesel production from mahua (Madhuca indica) oil using response surface methodology. Bioresour. Technol. 2006, 97, 379-384. [CrossRef]

87. Rodíguez, R.P.; Pérez, L.G.; Alfonso, M.; Duarte, M.; Caro, R.; Galle, J.; Sierens, R.; Verhelst, S. Thermal behavior of Jatropha curcas oils and their derived fatty acid ethyl esters as potential feedstocks for energy production in Cuba. J. Therm. Anal. Calorim. 2012, 109, 1005-1012. [CrossRef]

88. Canoira, L.; Alcántara, R.; Jesús García-Martínez, M.; Carrasco, J. Biodiesel from Jojoba oil-wax: Transesterification with methanol and properties as a fuel. Biomass Bioenergy 2006, 30, 76-81. [CrossRef]

89. Usta, N. Use of tobacco seed oil methyl ester in a turbocharged indirect injection diesel engine. Biomass Bioenergy 2005, 28, 77-86. [CrossRef]

90. Reyes, J.F.; Sepúlveda, M.A. PM-10 emissions and power of a Diesel engine fueled with crude and refined Biodiesel from salmon oil. Fuel 2006, 85, 1714-1719. [CrossRef]

91. Kansedo, J.; Lee, K.T.; Bhatia, S. Cerbera odollam (sea mango) oil as a promising non-edible feedstock for biodiesel production. Fuel 2009, 88, 1148-1150. [CrossRef]

92. Balat, M.; Balat, H. Progress in biodiesel processing. Appl. Energy 2010, 87, 1815-1835. [CrossRef]

93. Canakci, M. The potential of restaurant waste lipids as biodiesel feedstocks. Bioresour. Technol. 2007, 98, 183-190. [CrossRef] [PubMed]

94. Ma, F.; Hanna, M.A. Biodiesel production: A review. Bioresour. Technol. 1999, 70, 1-15. [CrossRef] 
95. Singh, S.P.; Singh, D. Biodiesel production through the use of different sources and characterization of oils and their esters as the substitute of diesel: A review. Renew. Sustain. Energy Rev. 2010, 14, 200-216. [CrossRef]

96. Hazrat, M.A.; Rasul, M.G.; Mofijur, M.; Khan, M.M.K.; Djavanroodi, F.; Azad, A.K.; Bhuiya, M.M.K.; Silitonga, A.S. A Mini Review on the Cold Flow Properties of Biodiesel and its Blends. Front. Energy Res. 2020, 8, 598651. [CrossRef]

97. Lang, X.; Dalai, A.K.; Bakhshi, N.N.; Reaney, M.J.; Hertz, P.B. Preparation and characterization of bio-diesels from various bio-oils. Bioresour. Technol. 2001, 80, 53-62. [CrossRef]

98. Patil, V.; Tran, K.Q.; Giselrød, H.R. Towards sustainable production of biofuels from microalgae. Int. J. Mol. Sci. 2008, 9, 1188-1195. [CrossRef]

99. Abomohra, A.E.-F.; Elsayed, M.; Esakkimuthu, S.; El-Sheekh, M.; Hanelt, D. Potential of fat, oil and grease (FOG) for biodiesel production: A critical review on the recent progress and future perspectives. Prog. Energy Combust. Sci. 2020, 81, 100868. [CrossRef]

100. Levine, B. New feedstock options and trends boost biodiesel production. Digest 2020, 5, 5-7.

101. Leong, W.-H.; Lim, J.-W.; Lam, M.-K.; Uemura, Y.; Ho, Y.-C. Third generation biofuels: A nutritional perspective in enhancing microbial lipid production. Renew. Sustain. Energy Rev. 2018, 91, 950-961. [CrossRef]

102. Deshmukh, S.; Kumar, R.; Bala, K. Microalgae biodiesel: A review on oil extraction, fatty acid composition, properties and effect on engine performance and emissions. Fuel Process. Technol. 2019, 191, 232-247. [CrossRef]

103. Sakthivel, R.; Ramesh, K.; Purnachandran, R.; Mohamed Shameer, P. A review on the properties, performance and emission aspects of the third generation biodiesels. Renew. Sustain. Energy Rev. 2018, 82, 2970-2992. [CrossRef]

104. Hemaiswarya, S.; Raja, R.; Carvalho, I.S.; Ravikumar, R.; Zambare, V.; Barh, D. An Indian scenario on renewable and sustainable energy sources with emphasis on algae. Appl. Microbiol. Biotechnol. 2012, 96, 1125-1135. [CrossRef]

105. Santana, A.; Jesus, S.; Larrayoz, M.A.; Filho, R.M. Supercritical carbon dioxide extraction of algal lipids for the biodiesel production. Procedia Eng. 2012, 42, 1755-1761. [CrossRef]

106. Ahmad, A.L.; Yasin, N.H.M.; Derek, C.J.C.; Lim, J.K. Microalgae as a sustainable energy source for biodiesel production: A review. Renew. Sustain. Energy Rev. 2011, 15, 584-593. [CrossRef]

107. Shah, S.H.; Raja, I.A.; Rizwan, M.; Rashid, N.; Mahmood, Q.; Shah, F.A.; Pervez, A. Potential of microalgal biodiesel production and its sustainability perspectives in Pakistan. Renew. Sustain. Energy Rev. 2018, 81, 76-92. [CrossRef]

108. Inganäs, O.; Sundström, V. Solar energy for electricity and fuels. Ambio 2016, 45, 15-23. [CrossRef]

109. Rabaey, K.; Rozendal, R.A. Microbial electrosynthesis-Revisiting the electrical route for microbial production. Nat. Rev. Microbiol. 2010, 8, 706-716. [CrossRef]

110. Gojun, M.; Pustahija, L.; Tušek, A.J.; Šalić, A.; Valinger, D.; Zelić, B. Kinetic parameter estimation and mathematical modelling of lipase catalysed biodiesel synthesis in a microreactor. Micromachines 2019, 10, 759. [CrossRef] [PubMed]

111. Ado, M.A.; Abas, F.; Mohammed, A.S.; Ghazali, H.M. Anti- and pro-lipase activity of selected medicinal, herbal and aquatic plants, and structure elucidation of an anti-lipase compound. Molecules 2013, 18, 14651-14669. [CrossRef] [PubMed]

112. Pallerla, O.; Vanitha, S. Isolation and Determination of Lipase activity in germinating Arachis hypogaea by Entrapment method Isolation and Determination of Lipase activity in germinating Arachis hypogaea by Entrapment method. Int. J. Sci. Res. Biol. Sci. 2019, 6, 142-147. [CrossRef]

113. Mittelbach, M. Lipase catalyzed alcoholysis of sunflower oil. J. Am. Oil Chem. Soc. 1990, 67, 168-170. [CrossRef]

114. Thakur, S. Lipases, Its sources, Properties and Applications: A Review. Int. J. Sci. Eng. Res. 2012, 3, 1-29.

115. Barros, M.; Fleuri, L.F.; MacEdo, G.A. Seed lipases: Sources, applications and properties-A review. Braz. J. Chem. Eng. 2010, 27, 15-29. [CrossRef]

116. Polizelli, P.P.; Facchini, F.D.A.; Bonilla-Rodriguez, G.O. Stability of a lipase extracted from seeds of pachira aquatica in commercial detergents and application tests in poultry wastewater pretreatment and fat particle hydrolysis. Enzym. Res. 2013, $2013,324061$. [CrossRef] [PubMed]

117. Ejedegba, B.O.; Onyeneke, E.C.; Oviasogie, P.O. Characteristics of lipase isolated from coconut (Cocos nucifera linn) seed under different nutrient treatments. Afr. J. Biotechnol. 2007, 6, 723-727.

118. Eastmond, P.J. Cloning and characterization of the acid lipase from Castor beans. J. Biol. Chem. 2004, 279, 45540-45545. [CrossRef]

119. Isbilir, S.S.; Ozcan, H.M.; Yagar, H. Some biochemical properties of lipase from bay laurel (Laurus nobilis L.) seeds. JAOCS J. Am. Oil Chem. Soc. 2008, 85, 227-233. [CrossRef]

120. Kanwar, R.; Mehta, D.K. Survey, collection and seed morphometric characterization of French bean (Phaseolus vulgaris L.) landraces of Himachal Pradesh. Legum. Res. 2018, 41, 333-341. [CrossRef]

121. Tuter, M.; Secundo, F.; Riva, S.; Ayşe Aksoy, H.; Ustun, G. Partial purification of Nigella sativa L. seed lipase and its application in transesterification reactions. J. Am. Oil Chem. Soc. 2003, 80, 43-48. [CrossRef]

122. Sana, N.K.; Hossin, I.; Haque, E.M.; Shaha, R.K. Identification, Purification and Characterization of Lipase from Germinating Oil Seeds (Brassica napus L.). Pak. J. Biol. Sci. 2004, 7, 246-252. [CrossRef]

123. Bhardwaj, K.; Raju, A.; Rajasekharan, R. Identification, purification, and characterization of a thermally stable lipase from rice bran. A new member of the (phospho) lipase family. Plant Physiol. 2001, 127, 1728-1738. [CrossRef] [PubMed]

124. Abigor, R.D.; Uadia, P.O.; Foglia, T.A.; Haas, M.J.; Scott, K.; Savary, B.J. Partial purification and properties of lipase from germinating seeds of Jatropha curcas L. J. Am. Oil Chem. Soc. 2002, 79, 1123-1126. [CrossRef] 
125. Borek, S.; Ratajczak, W.; Ratajczak, L. Ultrastructural and enzymatic research on the role of sucrose in mobilization of storage lipids in germinating yellow lupine seeds. Plant Sci. 2006, 170, 441-452. [CrossRef]

126. Al-Dhabi, N.A.; Esmail, G.A.; Ghilan, A.K.M.; Arasu, M.V. Isolation and screening of Streptomyces sp. Al-Dhabi-49 from the environment of Saudi Arabia with concomitant production of lipase and protease in submerged fermentation. Saudi J. Biol. Sci. 2020, 27, 474-479. [CrossRef] [PubMed]

127. Kumar, R.; Singh, D.; Swarnkar, M.K.; Singh, A.K.; Kumar, S. Genome assembly of Chryseobacterium polytrichastri ERMR1:04, a psychrotolerant bacterium with cold active proteases, isolated from east rathong glacier in India. Genome Announc. 2015, 3, 2014-2015. [CrossRef] [PubMed]

128. Kumar, A.; Mukhia, S.; Kumar, N.; Acharya, V.; Kumar, S.; Kumar, R. A Broad Temperature Active Lipase Purified from a Psychrotrophic Bacterium of Sikkim Himalaya with Potential Application in Detergent Formulation. Front. Bioeng. Biotechnol. 2020, 8, 642. [CrossRef]

129. Enujiugha, V.N.; Thani, F.A.; Sanni, T.M.; Abigor, R.D. Lipase activity in dormant seeds of the African oil bean (Pentaclethra macrophylla Benth). Food Chem. 2004, 88, 405-410. [CrossRef]

130. Pal, M.; Swarnkar, M.K.; Dhar, H.; Chhibber, S.; Gulati, A. Genome assembly of Chryseobacterium sp. strain IHBB 10212 from glacier top-surface soil in the Indian trans-Himalayas with potential for hydrolytic enzymes. Genom. Data 2017, 13, 46-49. [CrossRef]

131. Hassan, S.W.M.; El Latif, H.H.A.; Ali, S.M. Production of cold-active lipase by free and immobilized marine Bacillus cereus HSS: Application in wastewater treatment. Front. Microbiol. 2018, 9, 2377. [CrossRef] [PubMed]

132. Kai, W.; Peisheng, Y. Optimization of Lipase production from a novel strain Thalassospira permensis M35-15 using Response Surface Methodology. Bioengineered 2016, 7, 298-303. [CrossRef] [PubMed]

133. Saraswat, R.; Bhushan, I.; Gupta, P.; Kumar, V.; Verma, V. Production and purification of an alkaline lipase from Bacillus sp. for enantioselective resolution of $( \pm)$-Ketoprofen butyl ester. 3 Biotech 2018, 8, 491. [CrossRef] [PubMed]

134. Abol Fotouh, D.M.; Bayoumi, R.A.; Hassan, M.A. Production of Thermoalkaliphilic Lipase from Geobacillus thermoleovorans DA2 and Application in Leather Industry. Enzym. Res. 2016, 2016, 9034364. [CrossRef]

135. Biundo, A.; Hromic, A.; Pavkov-Keller, T.; Gruber, K.; Quartinello, F.; Haernvall, K.; Perz, V.; Arrell, M.S.; Zinn, M.; Ribitsch, D.; et al. Characterization of a poly(butylene adipate-co-terephthalate)-hydrolyzing lipase from Pelosinus fermentans. Appl. Microbiol. Biotechnol. 2016, 100, 1753-1764. [CrossRef]

136. Akbar, A.; Sitara, U.; Ali, I.; Muhammad, N.; Khan, S.A. Isolation and characterization of biotechnologically potent micrococcus luteus strain from environment. Pak. J. Zool. 2014, 46, 967-973.

137. Saun, N.K.; Narwal, S.K.; Dogra, P.; Chauhan, G.S.; Gupta, R. Comparative study of free and immobilized lipase from Bacillus aerius and its application in synthesis of ethyl ferulate. J. Oleo Sci. 2014, 63, 911-919. [CrossRef]

138. Yoo, H.Y.; Simkhada, J.R.; Cho, S.S.; Park, D.H.; Kim, S.W.; Seong, C.N.; Yoo, J.C. A novel alkaline lipase from Ralstonia with potential application in biodiesel production. Bioresour. Technol. 2011, 102, 6104-6111. [CrossRef] [PubMed]

139. Ülker, S.; Özel, A.; Çolak, A.; Alpay Karaoğlu, Ş. Isolation, production, and characterization of an extracellular lipase from Trichoderma harzianum isolated from soil. Turk. J. Biol. 2011, 35, 543-550. [CrossRef]

140. Uttatree, S.; Winayanuwattikun, P.; Charoenpanich, J. Isolation and characterization of a novel thermophilic-organic solvent stable lipase from Acinetobacter baylyi. Appl. Biochem. Biotechnol. 2010, 162, 1362-1376. [CrossRef]

141. Abdou, A.M. Purification and partial characterization of psychrotrophic Serratia marcescens lipase. J. Dairy Sci. 2003, 86, 127-132. [CrossRef]

142. Mehta, A.; Grover, C.; Bhardwaj, K.K.; Gupta, R. Application of lipase purified from aspergillus fumigatus in the syntheses of ethyl acetate and ethyl lactate. J. Oleo Sci. 2020, 69, 23-29. [CrossRef]

143. Silano, V.; Barat Baviera, J.M.; Bolognesi, C.; Cocconcelli, P.S.; Crebelli, R.; Gott, D.M.; Grob, K.; Lampi, E.; Mortensen, A.; Rivière, G.; et al. Safety evaluation of the food enzyme triacylglycerol lipase from Trichoderma reesei (strain RF10625). EFSA J. 2019, 17, e05837. [CrossRef]

144. Maldonado, R.R.; Lopes, D.B.; Aguiar-Oliveira, E.; Kamimura, E.S.; Macedo, G.A. A review on Geotrichum lipases: Production, purification, immobilization and applications. Chem. Biochem. Eng. Q. 2016, 30, 439-454. [CrossRef]

145. Gopinath, S.C.B.; Hilda, A.; Priya, T.L.; Annadurai, G. Purification of lipase from Cunninghamella verticillata and optimization of enzyme activity using response surface methodology. World J. Microbiol. Biotechnol. 2002, 18, 449-458. [CrossRef]

146. Toscano, L.; Gochev, V.; Montero, G.; Stoytcheva, M. Enhanced production of extracellular lipase by novel mutant strain of aspergillus niger. Biotechnol. Biotechnol. Equip. 2011, 25, 2243-2247. [CrossRef]

147. Teng, Y.; Xu, Y. Culture condition improvement for whole-cell lipase production in submerged fermentation by Rhizopus chinensis using statistical method. Bioresour. Technol. 2008, 99, 3900-3907. [CrossRef] [PubMed]

148. Ahammad, S.Z.; Gomes, J.; Sreekrishnan, T.R. Wastewater treatment forproductionofH2S-free biogas. J. Chem. Technol. Biotechnol. 2008, 83, 1163-1169. [CrossRef]

149. Sakpuntoon, V.; Angchuan, J.; Boontham, W.; Khunnamwong, P.; Boonmak, C.; Srisuk, N. Grease waste as a reservoir of lipase-producing yeast and description of Limtongella siamensis gen. Nov., sp. nov. Microorganisms 2020, 8, 27. [CrossRef] [PubMed] 
150. Yan, J.; Han, B.; Gui, X.; Wang, G.; Xu, L.; Yan, Y.; Madzak, C.; Pan, D.; Wang, Y.; Zha, G.; et al. Engineering Yarrowia lipolytica to Simultaneously Produce Lipase and Single Cell Protein from Agro-industrial Wastes for Feed. Sci. Rep. 2018, 8, 758. [CrossRef] [PubMed]

151. Çakmak, R.; Topal, G.; Çınar, E. Covalent Immobilization of Candida rugosa Lipase on Epichlorohydrin-Coated Magnetite Nanoparticles: Enantioselective Hydrolysis Studies of Some Racemic Esters and HPLC Analysis. Appl. Biochem. Biotechnol. 2020, 191, 1411-1431. [CrossRef] [PubMed]

152. Kapoor, M.; Majumder, A.B.; Gupta, M.N. Promiscuous Lipase-Catalyzed C-C Bond Formation Reactions between 4 Nitrobenzaldehyde and 2-Cyclohexen-1-one in Biphasic Medium: Aldol and Morita-Baylis-Hillman Adduct Formations. Catal. Lett. 2015, 145, 527-532. [CrossRef]

153. Del Pilar Rodriguez, M.; Brzezinski, R.; Faucheux, N.; Heitz, M. Enzymatic transesterification of lipids from microalgae into biodiesel: A review. AIMS Energy 2016, 4, 817-855. [CrossRef]

154. Šinkuniene, D.; Adlercreutz, P. Effects of regioselectivity and lipid class specificity of lipases on transesterification, exemplified by biodiesel production. J. Am. Oil Chem. Soc. 2014, 91, 1283-1290. [CrossRef]

155. João, J.H.; Tres, M.V.; Jahn, S.L.; de Oliveira, J.V. Lipases in liquid formulation for biodiesel production: Current status and challenges. Biotechnol. Appl. Biochem. 2020, 67, 648-667. [CrossRef]

156. Salwoom, L.; Rahman, R.N.Z.R.A.; Salleh, A.B.; Shariff, F.M.; Convey, P.; Ali, M.S.M. New recombinant cold-adapted and organic solvent tolerant lipase from psychrophilic Pseudomonas sp. Lsk25, isolated from signy island antarctica. Int. J. Mol. Sci. 2019, 20, 1264. [CrossRef]

157. Latip, W.; Raja Abd Rahman, R.N.Z.; Leow, A.T.C.; Mohd Shariff, F.; Kamarudin, N.H.A.; Mohamad Ali, M.S. The effect of N-terminal domain removal towards the biochemical and structural features of a thermotolerant lipase from an antarctic Pseudomonas sp. Strain AMS3. Int. J. Mol. Sci. 2018, 19, 560. [CrossRef] [PubMed]

158. De Almeida, A.F.; Tauk-Tornisielo, S.M.; Carmona, E.C. Acid lipase from Candida viswanathii: Production, biochemical properties, and potential application. BioMed Res. Int. 2013, 2013, 435818. [CrossRef]

159. Sethi, B.K.; Nanda, P.K.; Sahoo, S. Characterization of biotechnologically relevant extracellular lipase produced by Aspergillus terreus NCFT 4269.10. Braz. J. Microbiol. 2016, 47, 143-149. [CrossRef] [PubMed]

160. Kanmani, P.; Kumaresan, K.; Aravind, J. Gene cloning, expression, and characterization of the bacillus amyloliquefaciens PS35 lipase. Braz. J. Microbiol. 2015, 46, 1235-1243. [CrossRef] [PubMed]

161. Ali, N.S.M.; Salleh, A.B.; Rahman, R.N.Z.R.A.; Leow, T.C.; Ali, M.S.M. Calcium-induced activity and folding of a repeat in toxin lipase from antarctic pseudomonas fluorescens strain AMS8. Toxins 2020, 12, 27. [CrossRef] [PubMed]

162. Ilesanmi, O.I.; Adekunle, A.E.; Omolaiye, J.A.; Olorode, E.M.; Ogunkanmi, A.L. Isolation, optimization and molecular characterization of lipase producing bacteria from contaminated soil. Sci. Afr. 2020, 8, e00279. [CrossRef]

163. Tan, T.; Zhang, M.; Xu, J.; Zhang, J. Optimization of culture conditions and properties of lipase from Penicillium camembertii Thom PG-3. Process Biochem. 2004, 39, 1495-1502. [CrossRef]

164. Colen, G.; Junqueira, R.G.; Moraes-Santos, T. Isolation and screening of alkaline lipase-producing fungi from Brazilian savanna soil. World J. Microbiol. Biotechnol. 2006, 22, 881-885. [CrossRef]

165. Wu, J.P.; Li, M.; Zhou, Y.; Yang, L.R.; Xu, G. Introducing a salt bridge into the lipase of Stenotrophomonas maltophilia results in a very large increase in thermal stability. Biotechnol. Lett. 2015, 37, 403-407. [CrossRef]

166. Febriani; Aura, N.; Kemala, P.; Saidi, N.; Iqbalsyah, T.M. Novel thermostable lipase produced by a thermo-halophilic bacterium that catalyses hydrolytic and transesterification reactions. Heliyon 2020, 6, e04520. [CrossRef]

167. Arangio, A.M.; Slade, J.H.; Berkemeier, T.; Pöschl, U.; Knopf, D.A.; Shiraiwa, M. Multiphase chemical kinetics of oh radical uptake by molecular organic markers of biomass burning aerosols: Humidity and temperature dependence, surface reaction, and bulk diffusion. J. Phys. Chem. A 2015, 119, 4533-4544. [CrossRef]

168. Hasmah Ishak, S.N.; Ahmad Kamarudin, N.H.; Mohamad Ali, M.S.; Chor Leow, A.T.; Raja Noor Zaliha, R.N.Z.R. Ion-pair interaction and hydrogen bonds as main features of protein thermostability in mutated T1 recombinant lipase originating from Geobacillus zalihae. Molecules 2020, 25, 3430. [CrossRef]

169. Akoh, C.C.; Chang, S.W.; Lee, G.C.; Shaw, J.F. Enzymatic approach to biodiesel production. J. Agric. Food Chem. 2007, 55, 8995-9005. [CrossRef]

170. Wang, R.; Wang, S.; Xu, Y.; Yu, X. Enhancing the thermostability of Rhizopus chinensis lipase by rational design and MD simulations. Int. J. Biol. Macromol. 2020, 160, 1189-1200. [CrossRef]

171. Li, G.; Fang, X.; Su, F.; Chen, Y.; Xu, L.; Yan, Y. Enhancing the thermostability of Rhizomucor miehei lipase with a limited screening library by rational-design point mutations and disulfide bonds. Appl. Environ. Microbiol. 2018, 84, e02129-17. [CrossRef]

172. Zhao, J.; Wang, Z.; Gao, F.; Lin, J.; Yang, L.; Wu, M. Enhancing the thermostability of Rhizopus oryzae lipase by combined mutation of hot-spots and engineering a disulfide bond. RSC Adv. 2018, 8, 41247-41254. [CrossRef]

173. Christopher, L.P.; Zambare, V.P.; Zambare, A.; Kumar, H.; Malek, L. A thermo-alkaline lipase from a new thermophile Geobacillus thermodenitrificans AV-5 with potential application in biodiesel production. J. Chem. Technol. Biotechnol. 2015, 90, 2007-2016. [CrossRef]

174. Bhosale, H.; Shaheen, U.; Kadam, T. Characterization of a Hyperthermostable Alkaline Lipase from Bacillus sonorensis 4R. Enzym. Res. 2016, 2016, 4170684. [CrossRef] 
175. Sharma, R.; Soni, S.K.; Vohra, R.M.; Gupta, L.K.; Gupta, J.K. Purification and characterisation of a thermostable alkaline lipase from a new thermophilic Bacillus sp. RSJ-1. Process Biochem. 2002, 37, 1075-1084. [CrossRef]

176. Monteiro, R.R.C.; Lima, P.J.M.; Pinheiro, B.B.; Freire, T.M.; Dutra, L.M.U.; Fechine, P.B.A.; Gonçalves, L.R.B.; de Souza, M.C.M.; Dos Santos, J.C.S.; Fernandez-Lafuente, R. Immobilization of lipase a from Candida antarctica onto Chitosan-coated magnetic nanoparticles. Int. J. Mol. Sci. 2019, 20, 4018. [CrossRef] [PubMed]

177. Dalal, S.; Singh, P.K.; Raghava, S.; Rawat, S.; Gupta, M.N. Purification and properties of the alkaline lipase from Burkholderia cepacia A.T.C.C. 25609. Biotechnol. Appl. Biochem. 2008, 51, 23. [CrossRef]

178. Leow, T.C.; Rahman, R.N.Z.R.A.; Basri, M.; Salleh, A.B. A thermoalkaliphilic lipase of Geobacillus sp. T1. Extremophiles 2007, 11, 527-535. [CrossRef] [PubMed]

179. De Souza, W.; Reis, M.; Deda, R.; Ferreira, M.; Benedito, E. Immobilization of commercial lipase onto different supports: Characterization and application in esterification reaction. Int. J. Eng. Res. Sci. 2020, 6, 15-24.

180. Cardoso, C.L.; De Moraes, M.C.; Cass, Q.B. Imobilização de enzimas em suportes cromatográficos: Uma ferramenta na busca por substâncias bioativas. Quim. Nova 2009, 32, 175-187. [CrossRef]

181. Sun, J.; Chen, Y.; Sheng, J.; Sun, M. Immobilization of Yarrowia lipolytica Lipase on Macroporous Resin Using Different Methods: Characterization of the Biocatalysts in Hydrolysis Reaction. BioMed Res. Int. 2015, 2015, 139179. [CrossRef]

182. Khan, A.K.; Mubarak, N.M.; Abdullah, E.C.; Khalid, M.; Nizamuddin, S.; Baloch, H.A.; Siddiqui, M.T.H. Immobilization of Lipase Enzyme Carbon Nanotubes via Adsorption. IOP Conf. Ser. Mater. Sci. Eng. 2019, 495, 012055. [CrossRef]

183. Bajaj, A.; Lohan, P.; Jha, P.N.; Mehrotra, R. Biodiesel production through lipase catalyzed transesterification: An overview. J. Mol. Catal. B Enzym. 2010, 62, 9-14. [CrossRef]

184. He, Y.; Wang, X.; Wei, H.; Zhang, J.; Chen, B.; Chen, F. Direct enzymatic ethanolysis of potential Nannochloropsis biomass for co-production of sustainable biodiesel and nutraceutical eicosapentaenoic acid. Biotechnol. Biofuels 2019, 12, 78. [CrossRef]

185. Alavijeh, R.S.; Tabandeh, F.; Tavakoli, O.; Karkhane, A.; Shariati, P. Enzymatic production of biodiesel from microalgal oil using ethyl acetate as an acyl acceptor. J. Oleo Sci. 2015, 64, 69-74. [CrossRef]

186. Arumugam, A.; Ponnusami, V. Production of biodiesel by enzymatic transesterification of waste sardine oil and evaluation of its engine performance. Heliyon 2017, 3, e00486. [CrossRef]

187. Suhendra, D.; Gunawan, E.R.; Nurita, A.D.; Komalasari, D.; Ardianto, T. Optimization of the enzymatic synthesis of biodiesel from Terminalia cattapa L. Kernel oil using response surface methodology. J. Oleo Sci. 2017, 66, 209-215. [CrossRef]

188. Murillo, G.; He, Y.; Yan, Y.; Sun, J.; Bartocci, P.; Ali, S.S.; Fantozzi, F. Scaled-up biodiesel synthesis from Chinese Tallow Kernel oil catalyzed by Burkholderia cepacia lipase through ultrasonic assisted technology: A non-edible and alternative source of bio energy. Ultrason. Sonochem. 2019, 58, 104658. [CrossRef] [PubMed]

189. Choi, N.; Lee, J.S.; Kwak, J.; Lee, J.; Kim, I.H. Production of biodiesel from acid oil via a two-step enzymatic transesterification. J. Oleo Sci. 2016, 65, 913-921. [CrossRef]

190. Corrêa, I.N.D.S.; Lorena De Souza, S.; Catran, M.; Bernardes, O.L.; Portilho, M.F.; Langone, M.A.P. Enzymatic biodiesel synthesis using a byproduct obtained from palm oil refining. Enzym. Res. 2011, 2011, 814507. [CrossRef]

191. Yu, X.W.; Sha, C.; Guo, Y.L.; Xiao, R.; Xu, Y. High-level expression and characterization of a chimeric lipase from Rhizopus oryzae for biodiesel production. Biotechnol. Biofuels 2013, 6, 29. [CrossRef]

192. Kumari, A.; Mahapatra, P.; Garlapati, V.K.; Banerjee, R. Enzymatic transesterification of Jatropha oil. Biotechnol. Biofuels $2009,2,1$. [CrossRef]

193. Bhatti, H.N.; Hanif, M.A.; Qasim, M. Ata-ur-Rehman Biodiesel production from waste tallow. Fuel 2008, 87, 2961-2966. [CrossRef]

194. Surendhiran, D.; Sirajunnisa, A.R.; Vijay, M. An alternative method for production of microalgal biodiesel using novel Bacillus lipase. 3 Biotech 2015, 5, 715-725. [CrossRef] [PubMed]

195. Da Rós, P.C.M.; De Castro, H.F.; Carvalho, A.K.F.; Soares, C.M.F.; De Moraes, F.F.; Zanin, G.M. Microwave-assisted enzymatic synthesis of beef tallow biodiesel. J. Ind. Microbiol. Biotechnol. 2012, 39, 529-536. [CrossRef]

196. Pollardo, A.A.; Lee, H.S.; Lee, D.; Kim, S.; Kim, J. Solvent effect on the enzymatic production of biodiesel from waste animal fat. J. Clean. Prod. 2018, 185, 382-388. [CrossRef]

197. Lu, J.; Nie, K.; Xie, F.; Wang, F.; Tan, T. Enzymatic synthesis of fatty acid methyl esters from lard with immobilized Candida sp. 99-125. Process Biochem. 2007, 42, 1367-1370. [CrossRef]

198. Lee, K.T.; Foglia, T.A.; Chang, K.S. Production of alkyl ester as biodiesel from fractionated lard and restaurant grease. J. Am. Oil Chem. Soc. 2002, 79, 191-195. [CrossRef]

199. Huang, Y.; Zheng, H.; Yan, Y. Optimization of lipase-catalyzed transesterification of lard for biodiesel production using response surface methodology. Appl. Biochem. Biotechnol. 2010, 160, 504-515. [CrossRef]

200. Ali, A.; Kaur, M.; Mehra, U. Use of immobilized Pseudomonas sp. as whole cell catalyst for the transesterification of used cotton seed oil. J. Oleo Sci. 2011, 60, 7-10. [CrossRef]

201. Srimhan, P.; Kongnum, K.; Taweerodjanakarn, S.; Hongpattarakere, T. Selection of lipase producing yeasts for methanol-tolerant biocatalyst as whole cell application for palm-oil transesterification. Enzym. Microb. Technol. 2011, 48, 293-298. [CrossRef]

202. Xiao, M.; Qi, C.; Obbard, J.P. Biodiesel production using Aspergillus niger as a whole-cell biocatalyst in a packed-bed reactor. GCB Bioenergy 2011, 3, 293-298. [CrossRef] 
203. Pazouki, M.; Zamani, F.; Zamzamian, S.A.H.; Najafpour, G. Study on Reaction Conditions in Whole Cell Biocatalyst Methanolysis of Pretreated Used Cooking Oil. In Proceedings of the World Renewable Energy Congress-Sweden, Linköping, Sweden, 8-13 May 2011; Volume 57, pp. 93-100. [CrossRef]

204. Li, D.; Qin, X.; Wang, J.; Yang, B.; Wang, W.; Huang, W.; Wang, Y. Hydrolysis of soybean oil to produce diacylglycerol by a lipase from Rhizopus oryzae. J. Mol. Catal. B Enzym. 2015, 115, 43-50. [CrossRef]

205. Fior Markets. Global Lipase Market Is Expected to Reach USD 961.85 Million by 2028; Fior Market Research LLP: Pune, India, 2021.

206. Houde, A.; Kademi, A.; Leblanc, D. Lipases and Their Industrial Applications: An Overview. Appl. Biochem. Biotechnol. 2004, 118, 155-170. [CrossRef]

207. Fiormarkets Report. Lipase Market by Source (Animal Lipases, Microbial Lipases), Application (Animal Feed, Bakery, Dairy, Confectionery, Others), Region, Global Industry Analysis, Market Size, Share, Growth, Trends, and Forecast. 2021 to 2028; Fior Market Research LLP: Pune, India, 2021.

208. Rouhany, M.; Montgomery, H. Global Biodiesel Production: The State of the Art and Impact on Climate Change. In Biodiesel. Biofuel and Biorefinery Technologies; Tabatabaei, M., Aghbashlo, M., Eds.; Springer: Berlin/Heidelberg, Germany, 2019; pp. 1-14. ISBN 9783030009854.

209. Imark Report. Biodiesel Market: Global Industry Trends, Share, Size, Growth, Opportunity and Forecast 2021-2026; Imark Group: Noida, India, 2021.

210. Hobden, R. Commercializing enzymatic biodiesel production. Biodiesel Magazine, 3 January 2014.

211. Kotrba, R. SRS Int'l, biodiesel experts partner to offer enzymatic process. Biodiesel Magazine, 4 February 2015.

212. Kotrba, R. Buster biofuels to employ viesel fuel enzymatic biodiesel process. Biodiesel Magazine, 11 November 2014.

213. Kotrba, R. Blue Sun launches commercial-scale enzymatic biodiesel process. Biodiesel Magazine, 16 January 2014.

214. Tafes, A. Immobilized enzyme technology enters commercial stage. Biodiesel Magazine, 30 December 2014.

215. EnzymoCore's New Plant in Israel. Available online: https://enzymocore.com/news/transbiodiesels-new-plant-in-israel/ (accessed on 20 August 2021).

216. Tafesh, A. Biofuel International; Woodcote Media Ltd.: Morden, UK, 2015; pp. 44-45.

217. Aemetis-Inc. Aemetis completes enzymatic biodiesel production unit in India. Biodiesel Magazine, 28 August 2017.

218. Toldrá-Reig, F.; Mora, L.; Toldrá, F. Developments in the Use of Lipase Transesterification for Biodiesel Production from Animal Fat Waste. Appl. Sci. 2020, 10, 5085. [CrossRef]

219. Andreaus, J.; Camassola, M.; de Oliveira Faber, M.; Santana Ferreira-Leitão, V. ENZITEC special edition 2016 industrial applications of enzymes in Brazil. Biocatal. Biotransform. 2019, 37, 1-2. [CrossRef]

220. Mofijur, M.; Masjuki, H.H.; Kalam, M.A.; Atabani, A.E.; Fattah, I.M.R.; Mobarak, H.M. Comparative evaluation of performance and emission characteristics of Moringa oleifera and Palm oil based biodiesel in a diesel engine. Ind. Crop. Prod. 2014, 53, 78-84. [CrossRef]

221. Palash, S.M.; Masjuki, H.H.; Kalam, M.A.; Atabani, A.E.; Rizwanul Fattah, I.M.; Sanjid, A. Biodiesel production, characterization, diesel engine performance, and emission characteristics of methyl esters from Aphanamixis polystachya oil of Bangladesh. Energy Convers. Manag. 2015, 91, 149-157. [CrossRef]

222. Kumar, S.; Yadav, K.; Dwivedi, G. Impact analysis of oxidation stability for biodiesel \& its blends. Mater. Today Proc. 2018, 5, 19255-19261. [CrossRef]

223. Samuel, O.D.; Oreko, B.U.; Oyejide, J.O.; Idi, S.; Fayomi, O.S.I. Experimental and empirical study of diesel and biodiesel produced from blend of fresh vegetable and waste vegetable oil on density, viscosity, sulphur content and acid value. J. Phys. Conf. Ser. 2019, 1378, 042024. [CrossRef]

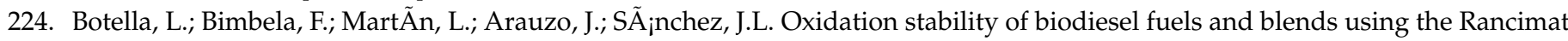
and PetroOXY methods. Effect of 4-allyl-2,6-dimethoxyphenol and catechol as biodiesel additives on oxidation stability. Front. Chem. 2014, 2, 43. [CrossRef]

225. Razzaq, L.; Farooq, M.; Mujtaba, M.A.; Sher, F.; Farhan, M.; Hassan, M.T.; Soudagar, M.E.M.; Atabani, A.E.; Kalam, M.A.; Imran, M. Modeling Viscosity and Density of Ethanol-Diesel-Biodiesel Ternary Blends for Sustainable Environment. Sustainability 2020, 12, 5186. [CrossRef]

226. Niculescu, R.; Clenci, A.; Iorga-Siman, V. Review on the use of diesel-biodiesel-alcohol blends in compression ignition engines. Energies 2019, 12, 1194. [CrossRef]

227. Ali, O.; Mamat, R.; Najafi, G.; Yusaf, T.; Safieddin Ardebili, S. Optimization of biodiesel-diesel blended fuel properties and engine performance with ether additive using statistical analysis and response surface methods. Energies 2015, 8, 14136-14150. [CrossRef]

228. Sazzad, B.S.; Fazal, M.A.; Haseeb, A.S.M.A.; Masjuki, H.H. Retardation of oxidation and material degradation in biodiesel: A review. RSC Adv. 2016, 6, 60244-60263. [CrossRef]

229. Velmurugan, K.; Sathiyagnanam, A.P. Impact of antioxidants on $\mathrm{NO} x$ emissions from a mango seed biodiesel powered DI diesel engine. Alex. Eng. J. 2016, 55, 715-722. [CrossRef]

230. Sierra-Cantor, J.F.; Guerrero-Fajardo, C.A. Methods for improving the cold flow properties of biodiesel with high saturated fatty acids content: A review. Renew. Sustain. Energy Rev. 2017, 72, 774-790. [CrossRef]

231. Bhuiya, M.; Rasul, M.; Khan, M.; Ashwath, N. Performance and Emission Characteristics of Binary Mixture of Poppy and Waste Cooking Biodiesel. Energy Procedia 2017, 110, 523-528. [CrossRef] 
232. Mahlia, T.M.I.; Syazmi, Z.A.H.S.; Mofijur, M.; Abas, A.E.P.; Bilad, M.R.; Ong, H.C.; Silitonga, A.S. Patent landscape review on biodiesel production: Technology updates. Renew. Sustain. Energy Rev. 2020, 118, 109526. [CrossRef]

233. Pradhan, A.; Mbohwa, C. Development of biofuels in South Africa: Challenges and opportunities. Renew. Sustain. Energy Rev. 2014, 39, 1089-1100. [CrossRef] 